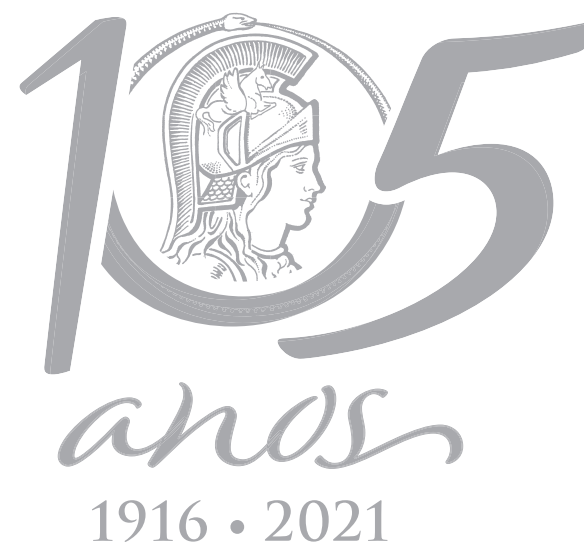

\title{
ECOSYSTEMS
}

\section{Insect galls and associated fauna in two areas of Cerrado sensu stricto in the State of Bahia, Brazil}

\author{
GABRIELA B.D. CAMPOS, ELAINE C. COSTA, DÉBORA L.S. SANTOS, SHEILA P. \\ CARVALHO-FERNANDES \& JULIANA SANTOS-SILVA
}

\begin{abstract}
This study inventoried and characterized the richness of galling insects based on gall morphotypes and their host plants in two Cerrado sensu stricto areas of Caetite municipality in Bahia State, Brazil, to aid the identification of galling insects and their host plants, as well as to contribute to the knowledge and conservation of local biodiversity. The survey was conducted in the Moita dos Porcos archaeological site and João Barroca Farm site, adopting the random walking methodology for sampling, during 12 months. We recorded 98 gall morphotypes on 42 plant species belonging to 36 genera and 22 families. Leguminosae, Malpighiaceae and Myrtaceae demonstrated the greatest richness of galls, and the Copaifera langsdorffii was the super-host species, with 16 gall morphotypes. Most galls occur on leaves, and may be globoid, glabrous, grouped, and are usually unilocular, and brown. The galling insects identified belonged to Coleoptera, Diptera, Lepidoptera, and Thysanoptera. Eight plant host species and eight gall morphotypes were recorded for the first time in Cerrado areas in Brazil. The areas surveyed demonstrated high richness of gall morphotypes and host plants, evidencing the importance of studying and preserving different areas of the same biome.
\end{abstract}

Key words: Copaifera galls, gall morphotypes, Malpighiaceae, savanna.

\section{INTRODUCTION}

Brazilian Cerrado is a hostpost of biodiversity, which is formed by mosaic of different phytophysiognomy, demonstrating elevated levels of species richness. It hosts more than 12,330 plant species (BFG 2018, 2021) and one third of Brazilian biodiversity, with a high level of endemism, making it the most biodiverse savanna in the world (Klink \& Machado 2005). In the state of Bahia, the Cerrado covers an area of approximately 9.1 million hectares (Faleiro 2015) distributed mainly in the western region of the state (Harley 1995). In addition, the Cerrado of Bahia is also distributed in higher regions forming small patches interspersed by Campos Rupestres, Caatinga and Seasonal Forests and transition areas, as occurs in Chapada Diamantina (Harley et al. 2005), and Serra Geral of the Caetité municipality (Bahia State, Brazil).

The Brazilian Cerrado is also one of the most threatened biomes, due to continuing deforestation. It is estimated that $43 \%$ of its native vegetation has already been removed or transformed (MMA 2015). The Cerrado areas of the Caetité municipality have been subjected to constant environmental impacts (Rodrigues et al. 2017) due to iron ore and limestone rock extraction (DNPM 2014), the construction of wind farms (Prudente et al. 2017), and amethyst mining.

Cerrado vegetation has several interactions among plants and herbivores (Ribeiro \& Fernandes 2000, Furtado et al. 2003, Cintra et al. 
2020). Among the different forms of herbivory, the endophytic habit of galling insects is by far the most sophisticated (Shorthouse et al. 2005). Galling insects are capable of inducing galls through disordered processes of hyperplasia, hypertrophy, and cellular differentiation in plant tissues (Ferreira \& Isaias 2013). The structures resulting from those interactions provide shelter for the gall-inducing insect against abiotic and biotic factors, allowing its successful development, and energetic resources for nutrition of the inducers (Price et al. 1987, Stone \& Schönrogge 2003).

The Brazilian Cerrado has been the subject of in-depth studies on the richness of galls, mainly in state of Minas Gerais (e.g., GonçalvesAlvim \& Fernandes 2001, Urso-Guimarães et al. 2003, Carneiro et al. 2009a, Araújo \& Guilherme 2012, Coelho et al. 2013, Cintra et al. 2020). The richness of gall studies in the Cerrado of Bahia State has been little studied; only five has yet been undertaken in the municipalities of Caetité (Nogueira et al. 2016, Vieira et al. 2018, Silva et al. 2018, Santana et al. 2020) and Barreiras (Lima \& Calado 2018). These studies in the Cerrado of Bahia recorded a total of 144 different types of galls in 103 plant species.

Studies of galling insect richness have demonstrated the usefulness of that insect guild as a measure of environmental conservation and quality (Santos et al. 2012, Santana \& Isaias 2014, Brito et al. 2018). The entomofauna associated with galls responds to environmental disturbances, with resulting losses of species richness and abundance (which also impact their natural enemies) (Oliveira 2009). As such, inventories of gall-inducing insect richness can assistance in urban planning, design, and management (Julião et al. 2005) as well as for environmental monitoring and conservation activities (Santana \& Isaias 2014, Melo-Júnior et al. 2018). Here, we realized an inventory of the richness of galling insects in two Cerrado sensu stricto areas of the Caetité municipality (Bahia) and a similarity analysis between areas based on gall morphotypes and their host plants to contribute to the knowledge and conservation of local biodiversity.

\section{MATERIALS AND METHODS}

\section{Study area}

The study was carried out in two localities of Cerrado sensu stricto vegetation in the municipality of Caetité, Bahia State, Brazil: João Barroca Farm (JB) (14²19'23.96" S and 42 33"7.91" W) situated about $40 \mathrm{~km}$ of Caetité's center, and the Moita dos Porcos archaeological site (MP) (14 $10^{\prime} 1.52^{\prime \prime} \mathrm{S}$ and $42^{\circ} 31^{\prime \prime} 16.05^{\prime \prime} \mathrm{W}$ ) approximately $15 \mathrm{~km}$ from the city center. The areas studied were selected on the basis of accessibility for sampling, the distance from previously sampled areas, and the presence of preserved vegetation (Costa et al. 2014a, b, Nogueira et al. 2016, Vieira et al. 2018, Silva et al. 2018, Santana et al. 2020). The JB comprises approximately 105 hectares of preserved area at $950 \mathrm{~m}$ elevation, while the MP covers approximately 300 hectares of preserved area at $920 \mathrm{~m}$ elevation. The vegetation is composed of many herbaceous and woody species, with a palm (Syagrus werdermannii Burret, Arecaceae) and sparse trees from 2 to $3 \mathrm{~m}$ tall (e.g., Hymenaea courbaril L., Leguminosae). The soil is sandy to sandy with rock outcrops on the higher elevation sites (Alves 2008). The regional climate is Aw (Alvares et al. 2013), with a mean annual temperature of $21.4^{\circ} \mathrm{C}$ and mean annual rainfall of $862 \mathrm{~mm}$ (IBGE 2015).

\section{Sampling method}

The Cerrado vegetation was examined during 12 months, between May 2016 to April 2017 throughout the MP site and between August 2017 to July 2018 at the JB site, adopting the random 
walking methodology for sampling (Julião et al. 2002, Oliveira \& Maia 2005, Coelho et al. 2009). In these areas, the galls and the host plants were collected once a month by two people working for four person-hours along pre-existing trails, totaling 96 hours of total collecting efforts. All plant architectural types (herbaceous, shrub, arboreal, tree and vines) up to $2 \mathrm{~m}$ tall were inspected. Each gall morphotype was photographed in field, and characterized based on shape, color, presence or absence of trichomes, number of internal chambers (Isaias et al. 2013). Specimens of the host plants were collected, pressed, and deposited in the Caetite collection of the HUNEB herbarium. The plants were identified by consulting the taxonomic literature and contacting botanical specialists, and by comparisons with herbarium collections at the Universidade do Estado da Bahia (HUNEB, Caetité collection) and the Universidade Estadual de Feira de Santana (HUEFS). Plant nomenclature was verified in the Flora do Brasil 2020 (www.floradobrasil.jbrj.gov. br) and, the names are presented in alphabetical order by family, following APG IV (2016). The circumscription of the Leguminosae family was based on classification proposed by LPWG (2017).

The samples of each gall morphotypes collected in the field were analyzed in laboratory. Galls were dissected under a stereomicroscope to retrieve any larvae of the gall-inducing insects and/or associated fauna, as well as observation the number of larval chamber. To obtain adults, samples of each gall morphotype were held separately in labeled plastic containers (lined with paper) and inspected daily. All of the insects obtained were conserved in $70 \%$ alcohol and sent to be identified at the Diptera Laboratory of the National Museum at the Universidade Federal do Rio de Janeiro.

\section{Statistical Analysis}

S $\varnothing$ rensen's similarity index $(S=2 c / a+b)$ (S $\phi$ rensen 1948) was used to compare the two localities in relation to the host plants ( $c=$ number of host plant species common to JB and MP, $\mathrm{a}=$ number of species in JB and $b=$ number of species in $\mathrm{MP}$ ) and gall morphotypes ( $\mathrm{c}=$ number of gall morphotypes common to JB and MP, $\mathrm{a}=$ number of gall morphotypes in JB and $b=$ number of gall morphotypes in MP).

\section{RESULTS}

We recorded 98 gall morphotypes on 42 plant species belonging to 36 genera and 22 families (Table I; Figures 1-5). Galls were recorded for the first time on the plant species Alchornea tiliifolia (Benth.) Müll. Arg. (Euphorbiaceae, Fig. 1m), Byrsonima correifolia A. Juss. (Malpighiaceae, Fig. 4C), Dalbergia acuta Benth. (LeguminoseaePapilionoideae, Fig. 3m), Erythroxylum stipulosum Plowman (Erythoxylaceae, Fig. 1l), Eugenia lucidifolia Barb. Rodr. (Myrtaceae, Fig. 5b), Pseudobrickellia brasiliensis (Spreng.) R. M. King \& Rob. (Asteraceae, Fig. 1h), Senna cana (Nees \& Mart.) H. S. Irwin \& Barneby var. cana (Leguminoseae- Caesalpinioideae, Fig. 2f), and Lippia origanoides Kunth (Verbenaceae, Fig. 5p).

A total of 73 gall morphotypes were found on the JB farm on 35 plant species belonging to 31 genera and 20 families (Table I). The species of the families Leguminosae, Malpighiaceae and Myrtaceae hosted the greatest gall richness, with 31, nine, and nine morphotypes, respectively. The plant genera with the greatest richness of gall morphotypes were Copaifera L. (LeguminosaeDetarioideae) ( $n=13, n=2$ host species) and Byrsonima Rich. ex Kunth (Malpighiaceae) ( $n=$ $7, n=4$ host species). The super-host species with the greatest richness of gall morphotypes 


\begin{tabular}{|c|c|c|c|c|c|c|c|c|c|c|c|c|c|c|c|c|c|c|c|c|c|}
\hline \multirow{2}{*}{\multicolumn{2}{|c|}{ 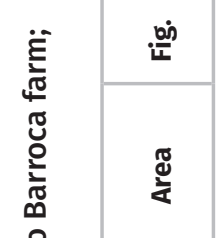 }} & & $\stackrel{\sigma}{\square}$ & $\stackrel{\circ}{\rightleftharpoons}$ & & $\stackrel{\cup}{\bullet}$ & $\stackrel{ }{-}$ & $\stackrel{\oplus}{-}$ & $\stackrel{\Psi}{\leftarrow}$ & & an & $\stackrel{\digamma}{\digamma}$ & & $:=$ & & $=$ & & 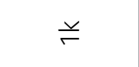 & $F$ & & $\xi$ \\
\hline & & & $\underline{\underline{ }}$ & $\stackrel{a}{\Sigma}$ & & $\stackrel{a}{\Sigma}$ & $\cong$ & $\begin{array}{l}\sum_{\Sigma}^{0} \\
\underline{\varrho}^{-}\end{array}$ & $\begin{array}{l}\sum_{\underline{\underline{m}}}^{0} \\
\underline{\underline{n}}\end{array}$ & & $\underline{\underline{n}}$ & $\underline{\underline{ }}$ & & $\underline{\underline{T}}$ & & $\begin{array}{l}\sum_{\varrho^{-}}^{0} \\
\underline{n}\end{array}$ & & $\begin{array}{l}\sum_{\infty}^{0} \\
\underline{\varrho}\end{array}$ & $\underline{\underline{ }}$ & & $\stackrel{\rho}{\Sigma}$ \\
\hline 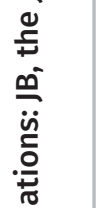 & 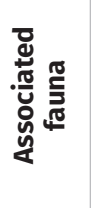 & & I & ' & & 1 & & 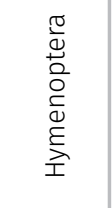 & , & & 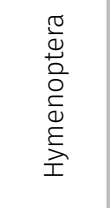 & ' & & , & & ' & & 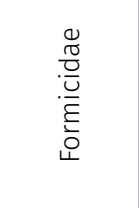 & & & ' \\
\hline 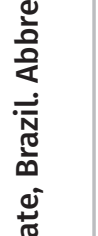 & 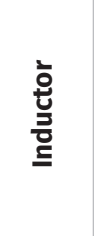 & & 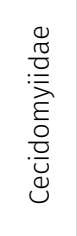 & , & & ' & ' & 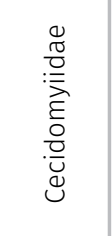 & ' & & 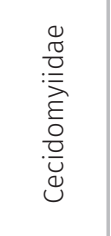 & ' & & ' & & 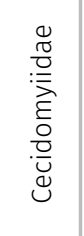 & & 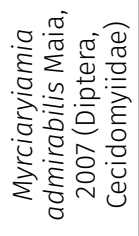 & & & , \\
\hline 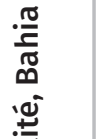 & 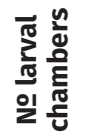 & & - & $r$ & & - & $r$ & $\begin{array}{l}\frac{n}{0} \\
\frac{0}{5} \\
\stackrel{5}{>}\end{array}$ & 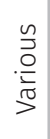 & & - & - & & - & & - & & $r$ & $r$ & & - \\
\hline $\begin{array}{l}\frac{4}{0} \\
\text { ते } \\
\frac{\vec{t}}{\pi}\end{array}$ & 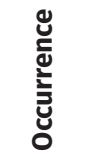 & & $\begin{array}{l}\overline{0} \\
0 \\
\frac{0}{7} \\
\overline{0} \\
\stackrel{0}{0}\end{array}$ & 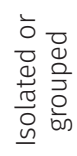 & & 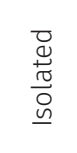 & $\begin{array}{l}\overline{0} \\
\stackrel{\mathbb{N}}{0} \\
\underline{0} \\
\underline{0}\end{array}$ & $\begin{array}{l}\overline{0} \\
\stackrel{0}{0} \\
\frac{0}{0} \\
\frac{0}{0}\end{array}$ & 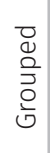 & & 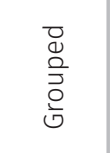 & $\begin{array}{l}\overline{0} \\
\stackrel{\mathbb{U}}{0} \\
\frac{\pi}{0} \\
\underline{0}\end{array}$ & & 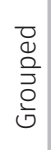 & & $\begin{array}{l}\overline{0} \\
\stackrel{0}{0} \\
\frac{\pi}{0} \\
\underline{0}\end{array}$ & & 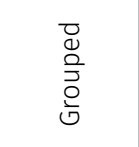 & $\begin{array}{l}0 \\
\stackrel{0}{0} \\
\underline{0} \\
\underline{0} \\
\underline{0}\end{array}$ & & $\begin{array}{l}\overline{0} \\
\stackrel{0}{0} \\
\frac{\pi}{0} \\
\underline{0}\end{array}$ \\
\hline 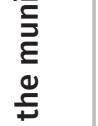 & $\begin{array}{l}\frac{\vec{n}}{\bar{n}} \\
\frac{0}{2}\end{array}$ & & $\stackrel{\circ}{z}$ & z & & zo & $\stackrel{\varrho}{\nu}$ & $\stackrel{\circ}{z}$ & z & & $\stackrel{\tilde{\Perp}}{\nsim}$ & z & & $\stackrel{0}{z}$ & & zo & & $\stackrel{\Perp}{\rightleftharpoons}$ & ㄹ & & 2) \\
\hline 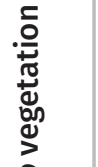 & $\begin{array}{l}\frac{\Perp}{\pi} \\
\frac{\pi}{n}\end{array}$ & & $\begin{array}{l}\frac{0}{0} \\
\frac{0}{0} \\
\frac{0}{0}\end{array}$ & 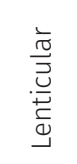 & & $\begin{array}{l}\frac{0}{0} \\
\frac{0}{0} \\
\frac{0}{0}\end{array}$ & $\begin{array}{l}\frac{0}{0} \\
\frac{0}{0} \\
\frac{0}{0}\end{array}$ & $\begin{array}{l}\frac{0}{0} \\
\frac{0}{0} \\
\frac{0}{0}\end{array}$ & $\begin{array}{l}\frac{n}{2} \\
\frac{0}{0} \\
\frac{0}{0} \\
\frac{\xi}{4}\end{array}$ & & $\begin{array}{l}\frac{0}{0} \\
\frac{0}{0} \\
\frac{0}{v}\end{array}$ & $\begin{array}{l}\frac{\overline{0}}{0} \\
\frac{0}{0} \\
\frac{0}{5}\end{array}$ & & $\begin{array}{l}\frac{n}{0} \\
\frac{0}{c} \\
\frac{0}{0} \\
\frac{c}{4}\end{array}$ & & $\begin{array}{l}\frac{0}{0} \\
\frac{0}{0} \\
\frac{0}{0}\end{array}$ & & $\begin{array}{l}\frac{0}{0} \\
\frac{0}{0} \\
\frac{0}{0}\end{array}$ & 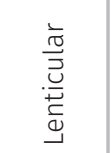 & & $\begin{array}{l}\frac{0}{0} \\
\frac{0}{0} \\
\frac{0}{0}\end{array}$ \\
\hline 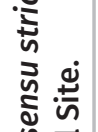 & $\frac{\text { 응 }}{3}$ & & 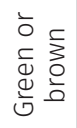 & 总 & & 疍 & 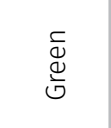 & $\begin{array}{l}\bar{\Phi} \\
\stackrel{\mathscr{U}}{\circlearrowleft}\end{array}$ & \begin{tabular}{l}
\multirow{3}{0}{} \\
$\stackrel{0}{\infty}$
\end{tabular} & & 䔂 & 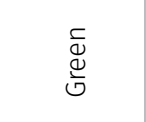 & & $\begin{array}{l}\frac{\ddot{u}}{\tilde{\omega}} \\
\frac{\pi}{0}\end{array}$ & & $\frac{z}{\partial}$ & & 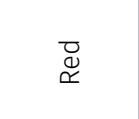 & 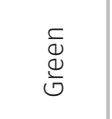 & & $\begin{array}{l}\stackrel{̃}{0} \\
\stackrel{0}{0}\end{array}$ \\
\hline 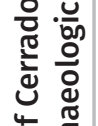 & $\underset{\widetilde{L}}{\mathscr{\Psi}}$ & & $\begin{array}{l}\bar{\sigma} \\
\frac{\pi}{x} \\
\mathbb{0} \\
\frac{0}{4}\end{array}$ & $\begin{array}{l}\frac{\overrightarrow{0}}{\frac{\pi}{x}} \\
\frac{\pi}{0}\end{array}$ & & & $\begin{array}{l}\frac{\vec{\pi}}{\sqrt{x}} \\
\frac{\pi}{\alpha}\end{array}$ & $\begin{array}{l}\frac{\pi}{\frac{\pi}{x}} \\
\frac{\pi}{0} \\
\frac{\pi}{4}\end{array}$ & $\begin{array}{l}\overrightarrow{.} \\
\frac{\pi}{x} \\
\frac{\pi}{0} \\
\frac{\pi}{4}\end{array}$ & & $\begin{array}{l}\frac{\vec{\pi}}{\pi} \\
\frac{\pi}{0} \\
\frac{\pi}{4}\end{array}$ & $\begin{array}{l}\overrightarrow{\frac{\pi}{0}} \\
\frac{\pi}{x} \\
\frac{\pi}{0}\end{array}$ & & & & $\begin{array}{l}\bar{\pi} \\
\frac{\pi}{x} \\
\frac{\pi}{0} \\
\frac{\pi}{4}\end{array}$ & & $\begin{array}{l}\overrightarrow{\frac{\pi}{x}} \\
\frac{\pi}{x} \\
\frac{\pi}{0}\end{array}$ & $\begin{array}{l}\overrightarrow{\frac{\pi}{0}} \\
\frac{\pi}{x} \\
\frac{\pi}{0}\end{array}$ & & 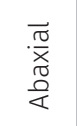 \\
\hline 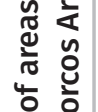 & $\begin{array}{l}\text { 。్ } \\
\text { ప్ }\end{array}$ & & 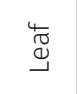 & 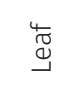 & & 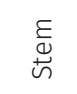 & 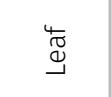 & 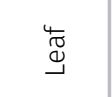 & 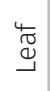 & & 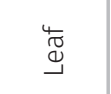 & $\begin{array}{l}E \\
\stackrel{E}{ \pm N}\end{array}$ & & 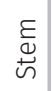 & & $\begin{array}{l}\stackrel{4}{\mathbb{J}} \\
\stackrel{\Xi}{\Xi}\end{array}$ & & 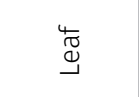 & 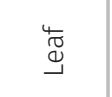 & & 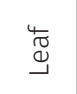 \\
\hline 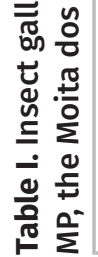 & 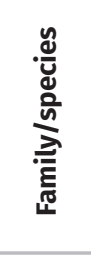 & 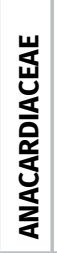 & 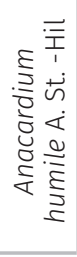 & & 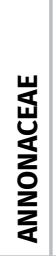 & 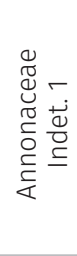 & 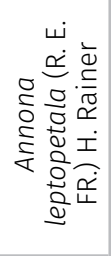 & 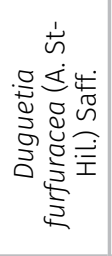 & & 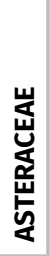 & 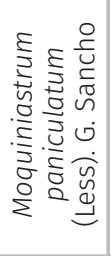 & 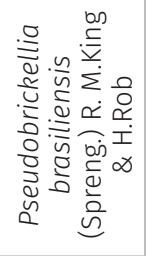 & 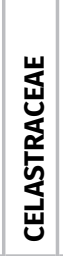 & 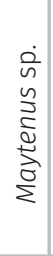 & 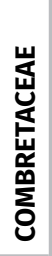 & 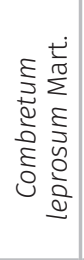 & 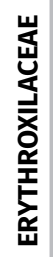 & 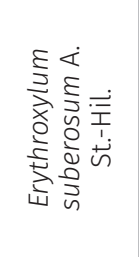 & 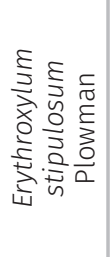 & 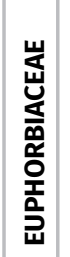 & 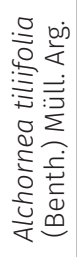 \\
\hline
\end{tabular}




\begin{tabular}{|c|c|c|c|c|c|c|c|c|c|c|c|c|c|c|c|c|c|c|c|c|c|}
\hline$\frac{a n g}{2}$ & $\subsetneq$ & & $\stackrel{\circ}{\circ}$ & & & 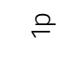 & $\check{\sigma}$ & 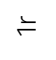 & $\cong$ & $F$ & ָే & $\stackrel{\sim}{\sim}$ & $\ddot{N}$ & $\stackrel{\nabla}{\sim}$ & $\stackrel{\sim}{\sim}$ & $\stackrel{4}{\sim}$ & & $\stackrel{b}{\sim}$ & 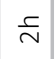 & $\bar{\sim}$ & $\bar{\sim}$ \\
\hline ®ூ & $\underline{\underline{T}}$ & & $\cong$ & & & $\begin{array}{l}\sum^{0} \\
\underline{\underline{0}}\end{array}$ & $\sum^{n}$ & $\underline{\underline{1}}$ & $\cong$ & $\cong$ & $\underline{\underline{1}}$ & $\underline{\underline{T}}$ & $\begin{array}{l}\sum_{\Sigma}^{n} \\
\varrho^{-}\end{array}$ & $\cong$ & $\underline{\varrho}$ & $\underline{\simeq}$ & & $\underline{\varrho}$ & 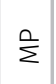 & $\underline{\varrho}$ & $\underline{\varrho}$ \\
\hline 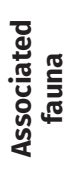 & & & & & & 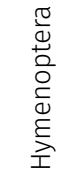 & ' & 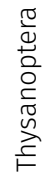 & 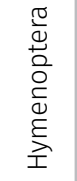 & $\begin{array}{l}\frac{0}{\frac{0}{\pi}} \\
\frac{0}{0} \\
\frac{0}{0} \\
\frac{0}{9}\end{array}$ & ' & & ' & ' & & ' & & & 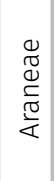 & ' & ' \\
\hline 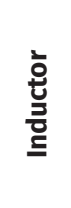 & 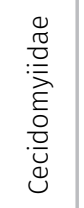 & & 1 & & & ' & ' & ' & ' & & 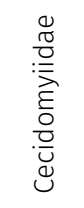 & 1 & 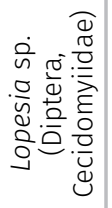 & 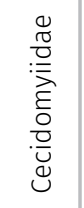 & ' & ' & & ' & ' & ' & $\begin{array}{l}\frac{0}{2} \\
\frac{0}{0} \\
\frac{0}{0} \\
\frac{0}{9}\end{array}$ \\
\hline 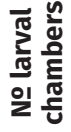 & $r$ & & $\frac{气}{\frac{n}{0}}$ & & & $r$ & $\frac{0}{\frac{0}{0}}$ & $r$ & - & $\begin{array}{l}\frac{n}{0} \\
\frac{0}{5} \\
\frac{0}{5}\end{array}$ & - & $r$ & $r$ & - & $\frac{气}{\frac{n}{0}}$ & $r$ & & $\begin{array}{l}\frac{n}{0} \\
\frac{0}{5} \\
\frac{10}{5}\end{array}$ & 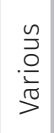 & 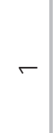 & - \\
\hline 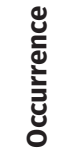 & $\begin{array}{l}\overline{0} \\
\stackrel{0}{0} \\
\overline{0} \\
\text { 임 }\end{array}$ & & $\begin{array}{l}\overline{0} \\
\stackrel{0}{0} \\
\underline{0} \\
\underline{0} \\
\underline{n}\end{array}$ & & & $\begin{array}{l}\overline{0} \\
0 \\
0 \\
\overline{0} \\
0 \\
0\end{array}$ & 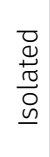 & $\begin{array}{l}\overline{0} \\
\stackrel{0}{0} \\
\frac{\pi}{0} \\
\underline{\underline{M}}\end{array}$ & $\begin{array}{l}\overline{0} \\
\underline{0} \\
\underline{0} 0 \\
\underline{0} \\
\underline{0}\end{array}$ & $\begin{array}{l}\overline{0} \\
\stackrel{ \pm}{0} \\
\frac{\pi}{0} \\
\underline{0}\end{array}$ & 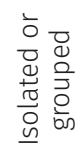 & $\begin{array}{l}\bar{d} \\
\stackrel{ \pm}{0} \\
\underline{0} \\
\underline{0}\end{array}$ & 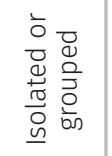 & 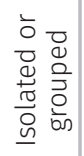 & $\begin{array}{l}\overline{0} \\
\stackrel{0}{0} \\
\frac{\pi}{0} \\
\underline{0}\end{array}$ & $\begin{array}{l}\overline{0} \\
\stackrel{ \pm}{0} \\
\underline{0} \\
\underline{0} \\
\underline{n}\end{array}$ & & $\begin{array}{l}\overline{0} \\
\stackrel{0}{0} \\
\frac{\pi}{0} \\
\underline{0}\end{array}$ & $\begin{array}{l}\text { D } \\
\stackrel{0}{7} \\
\frac{0}{0} \\
0\end{array}$ & $\begin{array}{l}\bar{g} \\
\stackrel{\mathbb{J}}{0} \\
\underline{0} \\
\underline{\underline{0}}\end{array}$ & 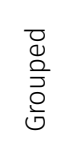 \\
\hline $\begin{array}{l}\frac{\vec{n}}{4} \\
\frac{0}{2}\end{array}$ & z & & 2 & & & zo & 2o & 20 & 을 & $\stackrel{\widetilde{J}}{\rightleftharpoons}$ & $\stackrel{\Perp}{\rightleftharpoons}$ & $\stackrel{\widetilde{a}}{\rightleftharpoons}$ & $\stackrel{\Perp}{\rightleftharpoons}$ & z & 2 & z & & z & $\stackrel{\varrho}{\infty}$ & 2 & 운 \\
\hline $\begin{array}{l}\frac{ \pm}{\pi} \\
\frac{\pi}{\omega}\end{array}$ & $\begin{array}{l}\xi \\
\frac{\xi}{0} \\
\frac{\stackrel{2}{n}}{\Psi} \\
\frac{1}{4}\end{array}$ & & $\begin{array}{l}\frac{0}{\circ} \\
\frac{8}{0} \\
\frac{0}{0}\end{array}$ & & & $\begin{array}{l}\frac{0}{8} \\
\frac{0}{0} \\
\frac{0}{0}\end{array}$ & 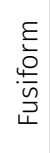 & 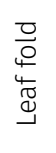 & 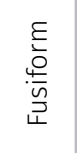 & $\begin{array}{l}\xi \\
\frac{\xi}{0} \\
\frac{\stackrel{2}{n}}{\Psi} \\
\frac{1}{4}\end{array}$ & 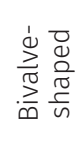 & 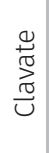 & 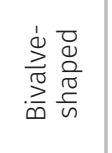 & 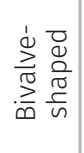 & $\begin{array}{l}\frac{0}{0} \\
\frac{0}{0} \\
\frac{0}{0}\end{array}$ & 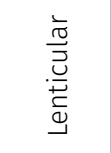 & & 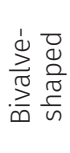 & $\begin{array}{l}\frac{0}{0} \\
\text { 응 } \\
\frac{0}{0}\end{array}$ & 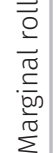 & 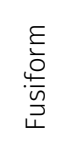 \\
\hline$\frac{\circ}{3}$ & 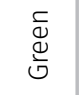 & & 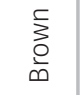 & & & $\begin{array}{l}\text { ב⿱ } \\
\frac{0}{0} \\
\end{array}$ & 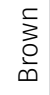 & 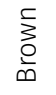 & 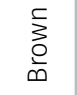 & 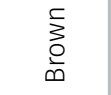 & 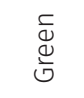 & $\begin{array}{l}\frac{1}{3} \\
\frac{0}{\infty}\end{array}$ & $\begin{array}{l}\frac{1}{0} \\
\frac{0}{0}\end{array}$ & $\begin{array}{l}\bar{\Phi} \\
\frac{\mathbb{d}}{\Delta}\end{array}$ & 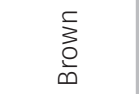 & $\begin{array}{l}\bar{q} \\
\propto\end{array}$ & & $\begin{array}{l}\frac{c}{\Phi} \\
\stackrel{\Phi}{\circlearrowleft}\end{array}$ & $\begin{array}{l}\stackrel{0}{3} \\
\frac{0}{\infty}\end{array}$ & $\begin{array}{l}\frac{\bar{Q}}{\Phi} \\
\stackrel{\mathbb{U}}{0}\end{array}$ & 竞 \\
\hline $\begin{array}{l}\mathscr{\Psi} \\
\mathbb{4}\end{array}$ & $\begin{array}{l}\frac{\vec{\sigma}}{x} \\
\frac{\pi}{0} \\
\frac{\pi}{4}\end{array}$ & & & & & ' & 1 & $\begin{array}{l}\frac{\pi}{\frac{\pi}{x}} \\
\frac{\pi}{\frac{\pi}{4}}\end{array}$ & ' & & $\begin{array}{l}\frac{\overrightarrow{0}}{\frac{\pi}{x}} \\
\frac{\pi}{\frac{\pi}{4}}\end{array}$ & $\begin{array}{l}\vec{\sigma} \\
\frac{\pi}{x} \\
\frac{\pi}{0} \\
\frac{0}{4}\end{array}$ & $\begin{array}{l}\frac{\bar{\sigma}}{\frac{\pi}{x}} \\
\frac{\pi}{\frac{\pi}{4}}\end{array}$ & $\begin{array}{l}\frac{\overrightarrow{0}}{\frac{\pi}{x}} \\
\frac{\pi}{\frac{\pi}{4}}\end{array}$ & & $\begin{array}{l}\frac{\overrightarrow{0}}{\frac{\pi}{\pi}} \\
\frac{\pi}{\alpha}\end{array}$ & & $\begin{array}{l}\bar{r} \\
\frac{\pi}{x} \\
\frac{\pi}{2}\end{array}$ & $\begin{array}{l}\frac{\overrightarrow{0}}{x} \\
\frac{\pi}{\frac{\pi}{\alpha}}\end{array}$ & , & ' \\
\hline 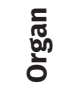 & $\begin{array}{l}\stackrel{4}{\mathbb{J}} \\
\end{array}$ & & $\begin{array}{l}\frac{E}{\Phi} \\
\stackrel{\Phi}{n}\end{array}$ & & $\frac{\underline{o}}{\underline{z}}$ & $\begin{array}{l}\frac{E}{\Phi} \\
\text { in }\end{array}$ & $\begin{array}{l}\frac{\varepsilon}{\Phi} \\
\text { in }\end{array}$ & $\stackrel{\sqrt[4]{\sigma}}{\Xi}$ & $\begin{array}{l}\frac{E}{\nu} \\
\text { 㟧 }\end{array}$ & $\begin{array}{l}E \\
\text { 焉 }\end{array}$ & 胥 & 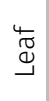 & & 胥 & $\begin{array}{l}E \\
\stackrel{E}{\Phi} \\
\dot{\omega}\end{array}$ & 胥 & $\begin{array}{l}\text { 岀 } \\
\text { 口о }\end{array}$ & 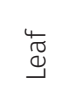 & 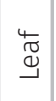 & $\begin{array}{l}\stackrel{4}{\mathbb{J}} \\
\stackrel{\Xi}{1}\end{array}$ & 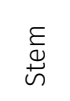 \\
\hline 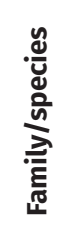 & 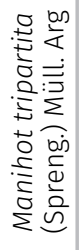 & 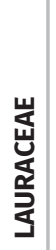 & 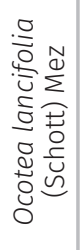 & 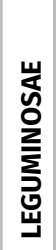 & 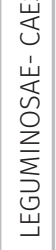 & 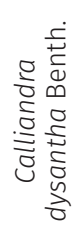 & & & 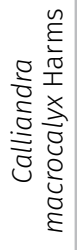 & 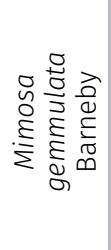 & & & & & 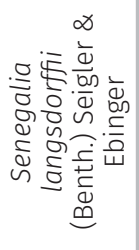 & 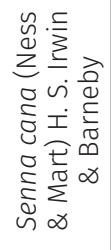 & 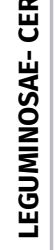 & 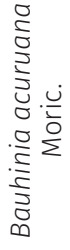 & & & 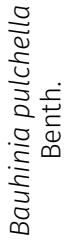 \\
\hline
\end{tabular}




\begin{tabular}{|c|c|c|c|c|c|c|c|c|c|c|c|c|c|c|c|c|c|c|c|c|c|c|c|c|c|c|}
\hline 夠 & $\stackrel{\ddot{N}}{ }$ & $\vec{\sim}$ & & $\underset{\sim}{\varepsilon}$ & $\lesssim$ & $\stackrel{\curvearrowright}{\sim}$ & $\stackrel{\circ}{\sim}$ & I & $\bar{i}$ & 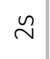 & $\stackrel{\sim}{\sim}$ & $\stackrel{\mathbb{N}}{m}$ & $\stackrel{\rho}{m}$ & $\ddot{m}$ & $\bar{m}$ & $\ddot{m}$ & $\stackrel{4}{m}$ & 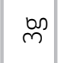 & $\frac{r}{m}$ & $\bar{m}$ & $\bar{m}$ & 弚 & & $\bar{m}$ & $\stackrel{\varepsilon}{\xi}$ & m \\
\hline 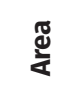 & $\underline{\underline{a}}$ & $\cong$ & & $\begin{array}{l}\sum_{\underline{\Sigma}}^{0} \\
\underline{0}\end{array}$ & 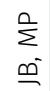 & 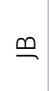 & $\stackrel{n}{\Sigma}$ & $\underline{\underline{ }}$ & 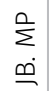 & $\varrho$ & $\begin{array}{l}\sum^{0} \\
\varrho^{-}\end{array}$ & $\begin{array}{l}\sum_{\varrho}^{n} \\
\varrho\end{array}$ & $\cong$ & $\underline{\underline{1}}$ & $\underline{\underline{1}}$ & $\cong$ & $\sum^{n}$ & $\bar{\Sigma}$ & $\bar{\Sigma}$ & $\stackrel{a}{\Sigma}$ & $\begin{array}{l}\sum_{\varrho}^{0} \\
\varrho^{-}\end{array}$ & $\underline{\underline{ }}$ & & $\sum_{\Sigma}^{0}$ & $\underline{\underline{ }}$ & $\underline{\underline{\prime}}$ \\
\hline 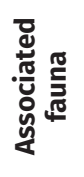 & , & ' & & 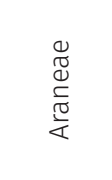 & 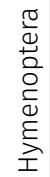 & & , & ' & 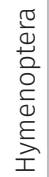 & ' & , & ' & & 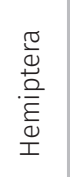 & ' & I & ' & ' & 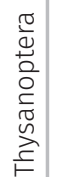 & ' & 1 & ' & & ' & ' & I \\
\hline $\begin{array}{l}\bar{t} \\
\underline{t} \\
\underline{\underline{\underline{t}}}\end{array}$ & 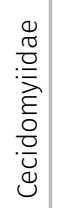 & 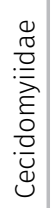 & & ' & ' & ' & 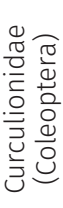 & ' & ' & ' & ' & ' & & 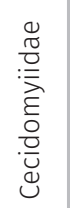 & 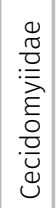 & ' & ' & ' & ' & ' & ' & I & & 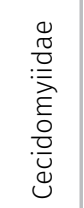 & ' & 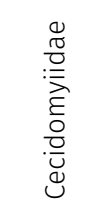 \\
\hline 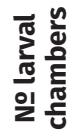 & - & $r$ & & $\frac{n}{\frac{n}{5}}$ & $r$ & $r$ & $\begin{array}{l}\frac{n}{0} \\
\frac{0}{n} \\
\stackrel{n}{>}\end{array}$ & $r$ & $r$ & - & $r$ & $\begin{array}{l}\frac{n}{3} \\
\frac{0}{\frac{0}{5}} \\
>\end{array}$ & - & $r$ & $r$ & $r$ & - & - & $r$ & - & $r$ & $r$ & & $r$ & $r$ & $r$ \\
\hline 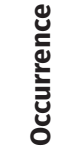 & $\begin{array}{l}\bar{Q} \\
\stackrel{0}{0} \\
\underline{0} \\
\underline{0}\end{array}$ & $\begin{array}{l}\overline{0} \\
\mathbb{0} \\
\frac{0}{7} \\
\frac{0}{v}\end{array}$ & & 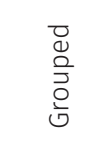 & $\begin{array}{l}\overline{0} \\
0 \\
\overline{0} \\
0 \\
0\end{array}$ & $\begin{array}{l}\overline{0} \\
\stackrel{d}{0} \\
\underline{0} \\
\underline{0} \\
\underline{n}\end{array}$ & $\begin{array}{l}\overline{0} \\
\stackrel{ \pm}{0} \\
\underline{0} \\
\underline{0}\end{array}$ & 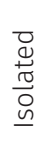 & $\begin{array}{l}\overline{0} \\
\stackrel{0}{0} \\
\text { 잉 }\end{array}$ & $\begin{array}{l}\overline{0} \\
\mathbb{d} \\
\bar{a} \\
\frac{0}{0} \\
\end{array}$ & $\begin{array}{l}\overline{0} \\
\mathbb{0} \\
\underline{0} \\
\underline{0} \\
\underline{M}\end{array}$ & $\begin{array}{l}\overline{0} \\
\stackrel{ \pm}{0} \\
\stackrel{0}{0} \\
\underline{0}\end{array}$ & 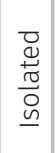 & 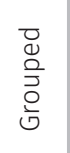 & 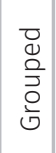 & $\begin{array}{l}\bar{g} \\
\stackrel{\mathbb{v}}{0} \\
\underline{0} \\
\underline{\underline{M}}\end{array}$ & $\begin{array}{l}\bar{D} \\
\stackrel{ \pm}{0} \\
\underline{0} \\
\underline{\underline{n}}\end{array}$ & $\mid \begin{array}{l}0 \\
\mathbb{d} \\
\underline{0} \\
0 \\
\underline{0} \\
\end{array}$ & $\begin{array}{l}\overrightarrow{0} \\
\stackrel{d}{0} \\
\underline{0} \\
\underline{0}\end{array}$ & 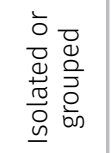 & 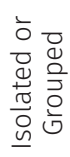 & $\begin{array}{l}\bar{g} \\
\underline{0} \\
\underline{0} \\
\underline{0}\end{array}$ & & 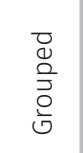 & 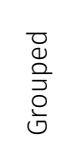 & $\begin{array}{l}\bar{D} \\
\stackrel{\mathbb{d}}{0} \\
\frac{\pi}{0} \\
\underline{\underline{n}}\end{array}$ \\
\hline $\begin{array}{l}\overrightarrow{\frac{1}{n}} \\
\text { 음 }\end{array}$ & $\stackrel{0}{z}$ & z & & $\stackrel{\circ}{z}$ & $\stackrel{\circ}{z}$ & zo & $\stackrel{o}{z}$ & 을 & $\stackrel{\widetilde{\jmath}}{\rightleftharpoons}$ & $\stackrel{o}{z}$ & z & z & $\stackrel{o}{z}$ & z & 2 & in & $\stackrel{0}{z}$ & z & i & $\stackrel{\circ}{z}$ & $\stackrel{\square}{\rightleftharpoons}$ & z & & $\stackrel{0}{z}$ & $\stackrel{\Perp}{\rightleftharpoons}$ & i \\
\hline $\begin{array}{l}\frac{\Xi}{\pi} \\
\frac{\pi}{n}\end{array}$ & 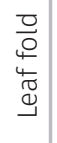 & $\begin{array}{l}\frac{0}{0} \\
\frac{0}{0} \\
\frac{0}{v}\end{array}$ & & 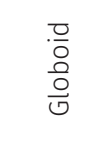 & $\begin{array}{l}\frac{.}{\overline{0}} \\
\text { 응 }\end{array}$ & 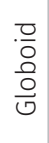 & $\begin{array}{l}\frac{\overline{0}}{0} \\
\frac{0}{0} \\
\frac{0}{v}\end{array}$ & 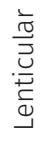 & $\begin{array}{l}\frac{\overline{0}}{\overline{0}} \\
\text { 응 }\end{array}$ & 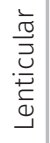 & $\begin{array}{l}\frac{\overline{0}}{0} \\
\frac{0}{0} \\
\frac{0}{0}\end{array}$ & $\begin{array}{l}\frac{\overline{0}}{0} \\
\frac{0}{0} \\
\frac{0}{v}\end{array}$ & 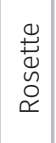 & 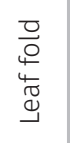 & $\begin{array}{l}\frac{0}{0} \\
\frac{8}{0} \\
\frac{0}{0}\end{array}$ & 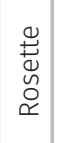 & 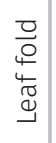 & $\mid \begin{array}{l}\frac{0}{0} \\
\frac{0}{0} \\
\frac{0}{0}\end{array}$ & $\begin{array}{l}\frac{\overline{0}}{0} \\
\frac{0}{0} \\
\frac{0}{v}\end{array}$ & 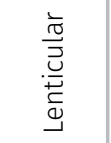 & $\begin{array}{l}\frac{0}{0} \\
\frac{0}{0} \\
\frac{0}{v}\end{array}$ & 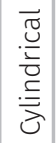 & & $\begin{array}{l}\varepsilon \\
\frac{g}{0} \\
\frac{0}{5} \\
\frac{g}{4}\end{array}$ & $\begin{array}{l}\overrightarrow{0} \\
\frac{\tilde{U}}{\sigma} \\
0\end{array}$ & $\begin{array}{l}\xi \\
\underline{0} \\
\frac{4}{5} \\
\xi\end{array}$ \\
\hline 흥 & 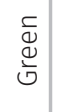 & 勇 & & 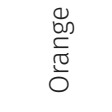 & 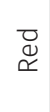 & 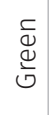 & 产 & 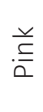 & $\begin{array}{l}\sum_{0}^{\complement} \\
\text { on }\end{array}$ & $\begin{array}{l}\frac{3}{0} \\
\frac{0}{\omega}\end{array}$ & $\begin{array}{l}\stackrel{c}{0} \\
\stackrel{0}{\infty}\end{array}$ & $\begin{array}{l}\bar{\Phi} \\
\stackrel{\Xi}{J} \\
\end{array}$ & $\begin{array}{l}\underset{\mathscr{Q}}{\alpha} \\
\text {. }\end{array}$ & $\begin{array}{l}\check{\Phi} \\
\stackrel{\Phi}{\cup}\end{array}$ & $\begin{array}{l}\vec{\otimes} \\
\propto\end{array}$ & 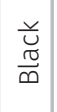 & $\begin{array}{l}0 \\
0 \\
\frac{0}{0} \\
0 \\
0\end{array}$ & $\begin{array}{l}\bar{q} \\
\propto \\
\propto\end{array}$ & $\begin{array}{l}\overrightarrow{\mathscr{x}} \\
\widetilde{x}\end{array}$ & $\begin{array}{l}\bar{d} \\
\stackrel{d}{J} \\
\end{array}$ & $\begin{array}{l}\stackrel{n}{0} \\
\stackrel{0}{0}\end{array}$ & $\begin{array}{l}\bar{\Phi} \\
\stackrel{\Xi}{\Xi} \\
\end{array}$ & & $\begin{array}{l}\bar{\Phi} \\
\stackrel{\Xi}{\Xi} \\
\end{array}$ & $\frac{3}{\bar{\theta}}$ & $\begin{array}{l}\stackrel{n}{0} \\
\stackrel{0}{0}\end{array}$ \\
\hline 芯 & I & $\begin{array}{l}\overrightarrow{.} \\
\frac{\pi}{x} \\
\frac{\pi}{0} \\
\frac{0}{\alpha}\end{array}$ & & 1 & ' & ' & , & $\begin{array}{l}\frac{\vec{\sigma}}{\frac{\pi}{x}} \\
\frac{\pi}{0}\end{array}$ & $\begin{array}{l}\frac{\bar{\sigma}}{\frac{\pi}{x}} \\
\frac{\pi}{\alpha}\end{array}$ & $\begin{array}{l}\frac{\pi}{\frac{\pi}{x}} \\
\frac{\pi}{\alpha} \\
\frac{\pi}{4}\end{array}$ & $\begin{array}{l}\frac{\pi}{\frac{\pi}{x}} \\
\frac{\pi}{\alpha} \\
\frac{⿱ 亠 乂}{4}\end{array}$ & $\begin{array}{l}\frac{\pi}{\frac{\pi}{x}} \\
\frac{\pi}{\alpha} \\
\frac{⿱ 亠 乂}{4}\end{array}$ & $\begin{array}{l}\overrightarrow{\bar{\sigma}} \\
\frac{\pi}{x} \\
\frac{\pi}{\alpha} \\
\frac{\pi}{2}\end{array}$ & 1 & $\begin{array}{l}\vec{\sigma} \\
\frac{\pi}{x} \\
\frac{\pi}{0} \\
\frac{0}{4}\end{array}$ & $\begin{array}{l}\overrightarrow{\frac{\pi}{x}} \\
\frac{\pi}{\frac{\pi}{4}}\end{array}$ & $\begin{array}{l}\vec{\pi} \\
\frac{\pi}{x} \\
\frac{\pi}{0} \\
\end{array}$ & $\mid \begin{array}{l}\vec{\pi} \\
\frac{\pi}{x} \\
\frac{\pi}{\alpha} \\
\end{array}$ & $\begin{array}{l}\frac{\vec{\sigma}}{x} \\
\frac{\pi}{\alpha}\end{array}$ & 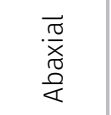 & $\begin{array}{l}\bar{\pi} \\
\frac{\pi}{x} \\
\frac{\pi}{4}\end{array}$ & $\begin{array}{l}\vec{\sigma} \\
\frac{\pi}{x} \\
\mathbb{0} \\
\frac{\mathbb{\alpha}}{\alpha}\end{array}$ & & 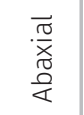 & ' & 1 \\
\hline $\begin{array}{l}\text { 苟 } \\
\text { to }\end{array}$ & 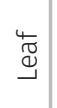 & 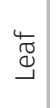 & $\begin{array}{l}\text { 岕 } \\
\text { 음 }\end{array}$ & 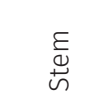 & $\begin{array}{l}\frac{E}{\Phi} \\
\stackrel{D}{\omega}\end{array}$ & $\begin{array}{l}E \\
\stackrel{\Xi}{\omega} \\
\dot{\omega n}\end{array}$ & 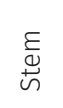 & $\stackrel{\sqrt[4]{\sigma}}{\Theta}$ & $\stackrel{4}{\mathbb{J}}$ & 离 & 离 & 嵻 & $\begin{array}{l}\stackrel{4}{\pi} \\
\stackrel{\Xi}{\Xi}\end{array}$ & $\begin{array}{l}\stackrel{4}{\pi} \\
\stackrel{\Xi}{\Xi}\end{array}$ & $\begin{array}{l}\stackrel{4}{\pi} \\
\stackrel{\Xi}{\leftrightarrows}\end{array}$ & $\begin{array}{l}\stackrel{4}{\sigma} \\
\Xi \\
\Xi\end{array}$ & 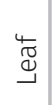 & $\begin{array}{l}\stackrel{4}{\sigma} \\
\stackrel{\Xi}{\Xi}\end{array}$ & 胥 & $\begin{array}{l}\stackrel{4}{\mathbb{J}} \\
\end{array}$ & 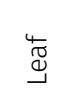 & 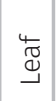 & $\begin{array}{l}\text { ô } \\
0 \\
\text { ô }\end{array}$ & $\begin{array}{l}\stackrel{4}{\pi} \\
\stackrel{\Xi}{\Theta}\end{array}$ & $\stackrel{\bar{D}}{\bar{D}}$ & 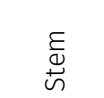 \\
\hline 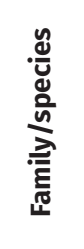 & & & 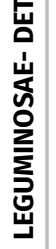 & 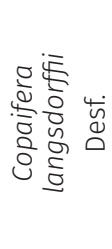 & & & & & & & & & & & & & & & & 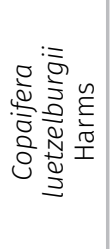 & 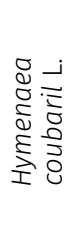 & & 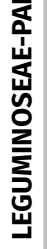 & 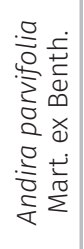 & 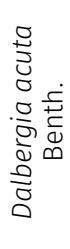 & 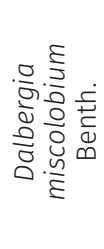 \\
\hline
\end{tabular}




\begin{tabular}{|c|c|c|c|c|c|c|c|c|c|c|c|c|c|c|c|c|c|c|c|c|c|}
\hline$\frac{b 3}{2}$ & & $\stackrel{\curvearrowright}{~}$ & & $\stackrel{\circ}{m}$ & $\bar{m}$ & $\bar{m}$ & $\tilde{m}$ & $\dot{m}$ & $\stackrel{\text { g }}{+}$ & f & y & ఫ্ণ & \& & 4 & $\underset{+}{\text { an }}$ & $\underset{f}{f}$ & $\bar{x}$ & 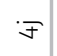 & $\stackrel{\check{y}}{\breve{y}}$ & $\vec{J}$ & \\
\hline 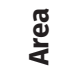 & & 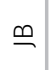 & & $\cong$ & $\underline{\varrho}$ & 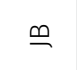 & $\bar{\Sigma}^{0}$ & $\sum_{\Sigma}^{0}$ & $\cong$ & $\underline{\underline{T}}$ & $\sum_{\Sigma}^{0}$ & $\cong$ & $\bar{\Sigma}$ & $\bar{\Sigma}$ & $\cong$ & $\sum^{n}$ & $\bar{\Sigma}$ & $\stackrel{a}{\Sigma}$ & $\underline{\underline{a}}$ & $\cong$ & \\
\hline 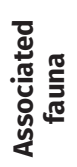 & & ' & & ' & & ' & & & & ' & 1 & ' & ' & ' & ' & ' & ' & 1 & ' & & \\
\hline 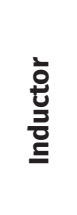 & & ' & & ' & ' & 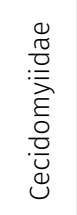 & ' & ' & $\begin{array}{l}\frac{0}{0} \\
\frac{0}{0} \\
\frac{0}{0} \\
\frac{0}{9}\end{array}$ & 1 & ' & 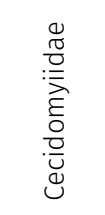 & ' & ' & ' & ' & ' & ' & ' & & \\
\hline 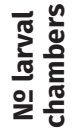 & & $r$ & & $r$ & $r$ & $r$ & $r$ & $r$ & $r$ & $\tau$ & - & $r$ & $r$ & $\begin{array}{l}\frac{n}{0} \\
\frac{0}{\sqrt{2}} \\
\frac{\sqrt{0}}{>}\end{array}$ & $r$ & - & - & - & $r$ & $r$ & \\
\hline 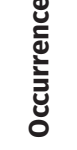 & & $\begin{array}{l}\bar{g} \\
\stackrel{d}{0} \\
\underline{0} \\
\underline{0} \\
\underline{n}\end{array}$ & & $\begin{array}{l}\bar{D} \\
\stackrel{0}{0} \\
\underline{0} \\
\underline{0} \\
\underline{0}\end{array}$ & 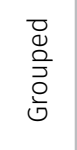 & $\begin{array}{l}\overline{0} \\
\stackrel{0}{0} \\
\overline{0} \\
0 \\
0\end{array}$ & $\begin{array}{l}\bar{d} \\
\stackrel{\mathbb{N}}{0} \\
\frac{\pi}{0} \\
\underline{0}\end{array}$ & $\begin{array}{l}\overline{0} \\
\stackrel{0}{0} \\
\overline{0} \\
\text { 입 }\end{array}$ & $\begin{array}{l}\bar{D} \\
\stackrel{0}{0} \\
\underline{0} \\
\underline{0} \\
\underline{0}\end{array}$ & $\begin{array}{l}\bar{d} \\
\stackrel{\underline{w}}{0} \\
\underline{0} \\
\underline{\underline{n}}\end{array}$ & 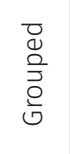 & $\begin{array}{l}\bar{d} \\
\stackrel{ \pm}{0} \\
\frac{\pi}{0} \\
\underline{\underline{M}}\end{array}$ & $\begin{array}{l}\overline{0} \\
\stackrel{0}{0} \\
\underline{0} \\
\underline{0} \\
\underline{0}\end{array}$ & $\begin{array}{l}\overline{0} \\
\stackrel{0}{0} \\
\frac{\pi}{0} \\
\underline{0}\end{array}$ & $\begin{array}{l}\overline{0} \\
\stackrel{0}{0} \\
\underline{0} \\
\underline{0}\end{array}$ & $\begin{array}{l}\overline{0} \\
\stackrel{ \pm}{0} \\
\frac{\pi}{0} \\
\underline{\underline{n}}\end{array}$ & $\begin{array}{l}\overline{0} \\
\stackrel{0}{0} \\
\underline{0} \\
\underline{0} \\
\underline{\underline{n}}\end{array}$ & $\begin{array}{l}\overline{0} \\
\stackrel{0}{0} \\
\underline{0} \\
\underline{0} \\
\underline{n}\end{array}$ & 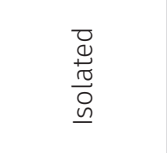 & 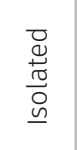 & \\
\hline $\begin{array}{l}\frac{\vec{े}}{\hat{n}} \\
\frac{0}{2}\end{array}$ & & $\stackrel{2}{z}$ & & $\stackrel{0}{z}$ & z & ㄹ & z & z & $\stackrel{\check{\nu}}{\rightleftharpoons}$ & 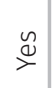 & z & 2 & $\stackrel{\check{\nu}}{\rightleftharpoons}$ & $\stackrel{\varrho}{\nu}$ & z & 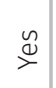 & $\frac{0}{2}$ & $\frac{0}{2}$ & $\stackrel{0}{z}$ & z & \\
\hline$\frac{\stackrel{ \pm}{\frac{0}{5}}}{\frac{\pi}{n}}$ & & 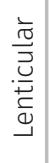 & & 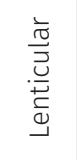 & . & 离 & $\begin{array}{l}\frac{}{\circ} \\
\frac{0}{0} \\
\frac{0}{0}\end{array}$ & 离 & $\begin{array}{l}\overline{\overline{0}} \\
\frac{\overline{0}}{\bar{\sigma}} \\
\frac{\bar{c}}{\mathrm{\omega}} \\
\frac{\bar{\sigma}}{2}\end{array}$ & $\begin{array}{l}\frac{0}{0} \\
\frac{0}{0} \\
\frac{0}{v}\end{array}$ & $\begin{array}{l}\frac{\overline{0}}{\circ} \\
\frac{8}{0} \\
\frac{0}{0}\end{array}$ & $\begin{array}{l}\frac{0}{8} \\
\frac{0}{0} \\
\frac{0}{0}\end{array}$ & $\begin{array}{l}\overrightarrow{\widetilde{\sigma}} \\
\stackrel{\widetilde{\sigma}}{\tilde{U}}\end{array}$ & $\begin{array}{l}\xi \\
\frac{\xi}{0} \\
\frac{0}{9} \\
\vec{\Psi}\end{array}$ & $\begin{array}{l}\frac{0}{0} \\
\frac{0}{0} \\
\frac{0}{5}\end{array}$ & 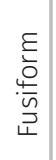 & $\begin{array}{l}\frac{\mathrm{D}}{\mathrm{O}} \\
\frac{\mathrm{a}}{\mathrm{O}} \\
\mathrm{v}\end{array}$ & $\begin{array}{l}\frac{0}{0} \\
\frac{0}{0} \\
\frac{0}{0}\end{array}$ & 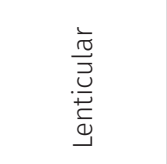 & 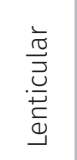 & \\
\hline 흥 & & 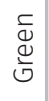 & & & 旁 & 竞 & 胥 & $\begin{array}{l}\text { בo } \\
\stackrel{0}{0}\end{array}$ & 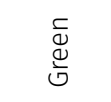 & 勇 & 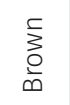 & 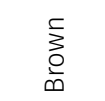 & $\begin{array}{l}\frac{c}{d} \\
\frac{d}{J}\end{array}$ & 离 & $\begin{array}{l}0 \\
\text { an } \\
\frac{0}{0} \\
\text { on }\end{array}$ & 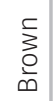 & $\begin{array}{l}\frac{n}{3} \\
\frac{0}{0}\end{array}$ & 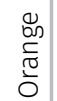 & 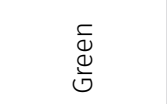 & $\stackrel{\text { 兰 }}{a}$ & \\
\hline 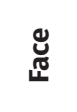 & & $\begin{array}{l}\frac{\vec{\pi}}{\sqrt{x}} \\
\frac{\pi}{\alpha} \\
\frac{0}{4}\end{array}$ & & $\begin{array}{l}\frac{\vec{\pi}}{\frac{\pi}{x}} \\
\frac{\pi}{0} \\
\frac{\pi}{4}\end{array}$ & $\begin{array}{l}\frac{\vec{\pi}}{\pi} \\
\frac{\pi}{\pi} \\
\frac{\pi}{4}\end{array}$ & $\begin{array}{l}\frac{\vec{\pi}}{\frac{\pi}{x}} \\
\frac{\pi}{0} \\
\frac{\pi}{4}\end{array}$ & $\begin{array}{l}\frac{\vec{\pi}}{\pi} \\
\frac{\pi}{\pi} \\
\frac{\pi}{4}\end{array}$ & $\begin{array}{l}\frac{\bar{\pi}}{x} \\
\frac{\pi}{\frac{\pi}{4}}\end{array}$ & & ' & ' & $\begin{array}{l}\frac{\pi}{\frac{\pi}{x}} \\
\frac{\pi}{\frac{\pi}{4}}\end{array}$ & $\begin{array}{l}\frac{\overrightarrow{0}}{\pi} \\
\frac{\pi}{\pi} \\
\frac{\pi}{4}\end{array}$ & ' & $\begin{array}{l}\frac{\vec{\pi}}{x} \\
\frac{\pi}{0} \\
\frac{0}{4}\end{array}$ & $\begin{array}{l}\overrightarrow{\frac{\pi}{x}} \\
\frac{\pi}{x} \\
\frac{\pi}{4}\end{array}$ & , & $\begin{array}{l}\overline{\frac{\pi}{x}} \\
\frac{\pi}{\pi} \\
\frac{\pi}{4}\end{array}$ & $\begin{array}{l}\frac{\pi}{\frac{\pi}{x}} \\
\frac{\pi}{\frac{\pi}{4}}\end{array}$ & $\begin{array}{l}\frac{\vec{\pi}}{\frac{\pi}{x}} \\
\frac{\pi}{\frac{\pi}{4}}\end{array}$ & \\
\hline $\begin{array}{l}\text { 苟 } \\
\text { ప }\end{array}$ & & 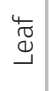 & & 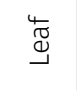 & 离 & 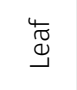 & $\stackrel{4}{\mathbb{J}}$ & 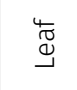 & $\stackrel{\stackrel{4}{\mathbb{J}}}{{ }_{-1}^{*}}$ & $\stackrel{\mathbb{J}}{\mathbb{\Xi}}$ & 离 & 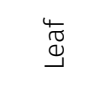 & 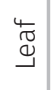 & $\begin{array}{l}E \\
\stackrel{E}{\Phi} \\
心\end{array}$ & $\begin{array}{l}\overrightarrow{0} \\
\vec{D}\end{array}$ & 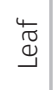 & 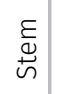 & 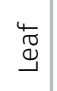 & 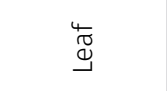 & 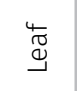 & \\
\hline 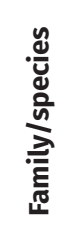 & 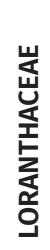 & 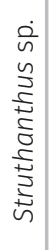 & 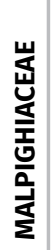 & 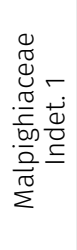 & 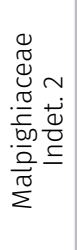 & 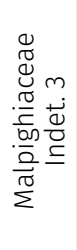 & 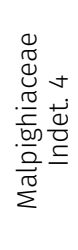 & 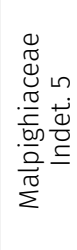 & 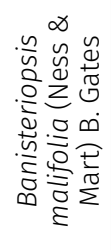 & & 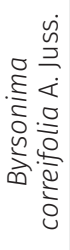 & 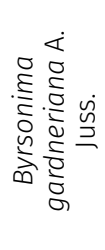 & & 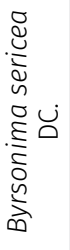 & & & 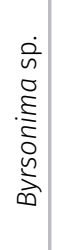 & 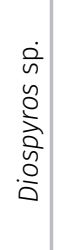 & 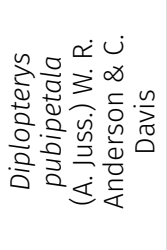 & 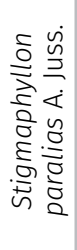 & 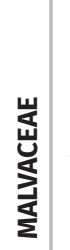 \\
\hline
\end{tabular}




\begin{tabular}{|c|c|c|c|c|c|c|c|c|c|c|c|c|c|c|c|c|c|c|c|c|c|}
\hline$\frac{b j}{5}$ & & 乎 & 워 & & ? & F & 于 & 字 & 莳 & డే & 느 & 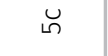 & 뮤 & $\stackrel{\unrhd}{n}$ & 㭊 & 占 & & है & & in & $i \bar{n}$ \\
\hline 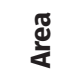 & & $\underline{\simeq}$ & $\underline{\underline{ }}$ & & $\bar{\Sigma}$ & $\cong$ & $\bar{\Sigma}$ & 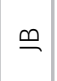 & $\unrhd$ & 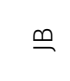 & $\bar{\Sigma}$ & $\cong$ & $\underline{\underline{ }}$ & $\cong$ & $\cong$ & $\underline{\underline{ }}$ & & $\sum_{\Sigma}^{0}$ & & $\cong$ & $\sum_{\underline{\underline{a}}}^{0}$ \\
\hline 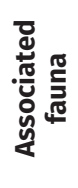 & & ' & ' & & ' & ' & ' & ' & ' & 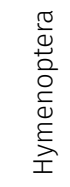 & 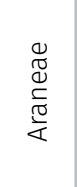 & 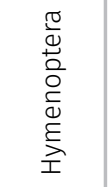 & ' & ' & ' & ' & & 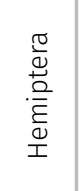 & & & 1 \\
\hline 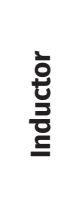 & & ' & ' & & ' & ' & ' & ' & ' & 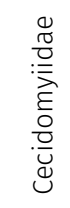 & 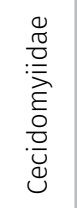 & ' & ' & ' & ' & 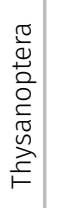 & & 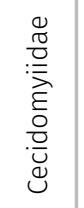 & & ' & 1 \\
\hline 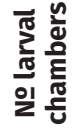 & & $r$ & $r$ & & 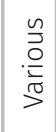 & - & $\mid$\begin{tabular}{l|}
$\frac{n}{3}$ \\
.$\frac{0}{5}$ \\
$\frac{5}{5}$
\end{tabular} & $\tau$ & $r$ & - & - & 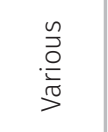 & $r$ & - & $r$ & - & & $r$ & & $r$ & - \\
\hline 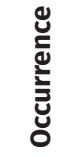 & & 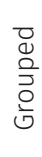 & $\begin{array}{l}\bar{D} \\
\stackrel{d}{0} \\
\stackrel{0}{0} \\
\underline{n}\end{array}$ & & 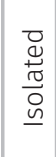 & $\begin{array}{l}0 \\
\stackrel{0}{0} \\
\underline{0} \\
0 \\
\underline{n}\end{array}$ & 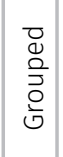 & 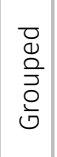 & $\begin{array}{l}\overline{0} \\
\stackrel{0}{0} \\
\underline{0} \\
\underline{0} \\
\underline{n}\end{array}$ & $\begin{array}{l}\overline{0} \\
\stackrel{0}{0} \\
\frac{\pi}{0} \\
\underline{0}\end{array}$ & 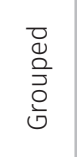 & 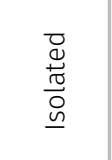 & $\begin{array}{l}\bar{d} \\
\underline{\mathbb{d}} \\
\underline{0} \\
\underline{\underline{n}}\end{array}$ & $\begin{array}{l}\overline{0} \\
\stackrel{0}{0} \\
\underline{0} \\
\underline{0} \\
\underline{0}\end{array}$ & 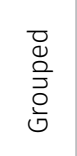 & $\begin{array}{l}\overline{0} \\
0 \\
\frac{0}{7} \\
\frac{0}{0} \\
\text { d. }\end{array}$ & & 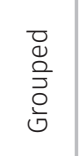 & & 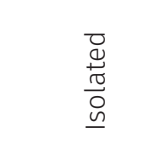 & $\begin{array}{l}\overline{0} \\
\stackrel{0}{0} \\
\underline{0} \\
\underline{0}\end{array}$ \\
\hline $\begin{array}{l}\overrightarrow{\frac{\vec{n}}{n}} \\
\frac{0}{0}\end{array}$ & & 우 & 20 & & 2 & z & $\stackrel{\mathscr{\Xi}}{\nu}$ & 2 & $\stackrel{0}{z}$ & $\stackrel{\circ}{z}$ & 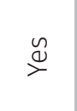 & $\stackrel{\circ}{z}$ & $\stackrel{0}{z}$ & $\stackrel{\varrho}{\rightleftharpoons}$ & 2 & $\stackrel{0}{2}$ & & z & & ㅇ & $\stackrel{o}{z}$ \\
\hline 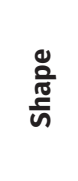 & & 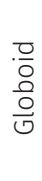 & 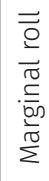 & & $\begin{array}{l}\frac{\overline{0}}{\overline{0}} \\
\frac{0}{0} \\
0\end{array}$ & 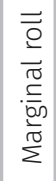 & $\begin{array}{l}: 0 \\
0 \\
\frac{0}{0} \\
0 \\
0\end{array}$ & 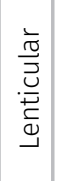 & $\begin{array}{l}\frac{}{0} \\
\frac{0}{0} \\
\frac{0}{0}\end{array}$ & 离 & $\begin{array}{l}\frac{\bar{\sigma}}{\pi} \\
\frac{0}{0}\end{array}$ & 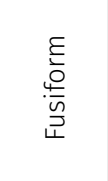 & 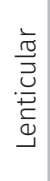 & 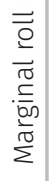 & $\begin{array}{l}\frac{}{0} \\
\frac{0}{0} \\
\frac{0}{0}\end{array}$ & 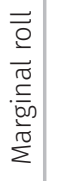 & & 克 & & 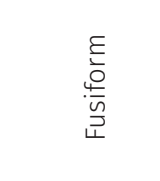 & 克 \\
\hline 흥 & & $\sum_{\frac{0}{0}}^{\frac{5}{0}}$ & 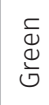 & & 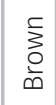 & 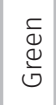 & $\begin{array}{l}\underset{\otimes}{\mathscr{Q}} \\
\end{array}$ & $\begin{array}{l}\overline{\mathscr{Q}} \\
\propto\end{array}$ & $\underset{\frac{3}{0}}{\tilde{y}}$ & 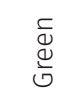 & $\begin{array}{l}\ddot{\otimes} \\
\propto\end{array}$ & 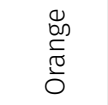 & $\begin{array}{l}\frac{\ddot{0}}{\tilde{\omega}} \\
\frac{\pi}{0}\end{array}$ & $\stackrel{\text { 咅 }}{\text {. }}$ & $\begin{array}{l}\frac{\ddot{U}}{\frac{\pi}{\infty}} \\
\frac{1}{0}\end{array}$ & 美 & & 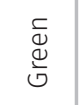 & & 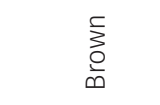 & 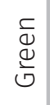 \\
\hline 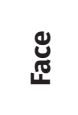 & & & I & & ' & ' & $\begin{array}{l}\overrightarrow{\frac{\pi}{x}} \\
\frac{\pi}{\pi} \\
\frac{\pi}{4}\end{array}$ & 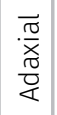 & ' & $\begin{array}{l}\frac{\bar{\pi}}{\frac{\pi}{x}} \\
\frac{\pi}{\frac{\pi}{4}}\end{array}$ & $\begin{array}{l}\frac{\bar{\pi}}{x} \\
\frac{\pi}{\frac{\pi}{4}}\end{array}$ & & $\begin{array}{l}\frac{\vec{\pi}}{\frac{\pi}{x}} \\
\frac{\pi}{\frac{\pi}{4}}\end{array}$ & . & ' & 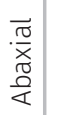 & & $\begin{array}{l}\frac{\pi}{\pi} \\
\frac{\pi}{\pi} \\
\frac{\pi}{2}\end{array}$ & & & $\begin{array}{l}\overrightarrow{\frac{\pi}{x}} \\
\frac{\pi}{\pi} \\
\frac{\pi}{x}\end{array}$ \\
\hline 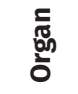 & & $\begin{array}{l}E \\
\stackrel{\Xi}{ \pm} \\
\dot{\omega}\end{array}$ & 崖 & & $\begin{array}{l}\varepsilon \overline{ \pm} \\
\stackrel{\Xi}{\omega}\end{array}$ & 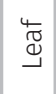 & \begin{tabular}{|l|}
$\stackrel{4}{\mathbb{J}}$ \\
$\mathbb{G}$
\end{tabular} & $\begin{array}{l}\sqrt[4]{\pi} \\
\stackrel{\Xi}{\Xi}\end{array}$ & $\begin{array}{l}E \\
\text { 焉 }\end{array}$ & 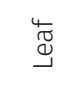 & $\begin{array}{l}\sqrt[\pi]{9} \\
\stackrel{9}{9}\end{array}$ & ב. & 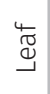 & $\stackrel{4}{\mathbb{J}}$ & $\stackrel{\bar{\partial}}{\overrightarrow{0}}$ & 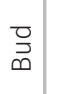 & & 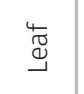 & & $\begin{array}{l}\frac{E}{\Phi} \\
\stackrel{ \pm}{\omega}\end{array}$ & $\begin{array}{l}\stackrel{4}{\widetilde{\Xi}} \\
\Xi\end{array}$ \\
\hline 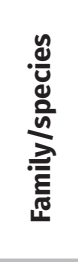 & 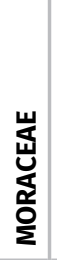 & 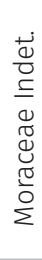 & & 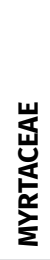 & 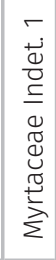 & 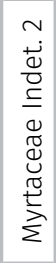 & 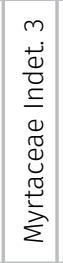 & 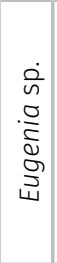 & 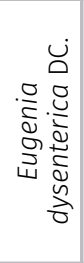 & & 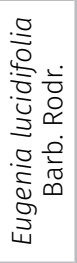 & 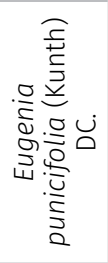 & & & 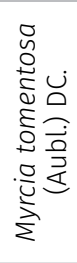 & & בù & 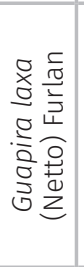 & 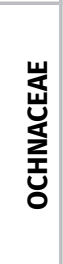 & 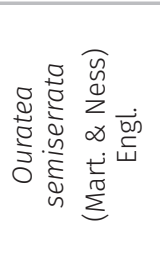 & \\
\hline
\end{tabular}




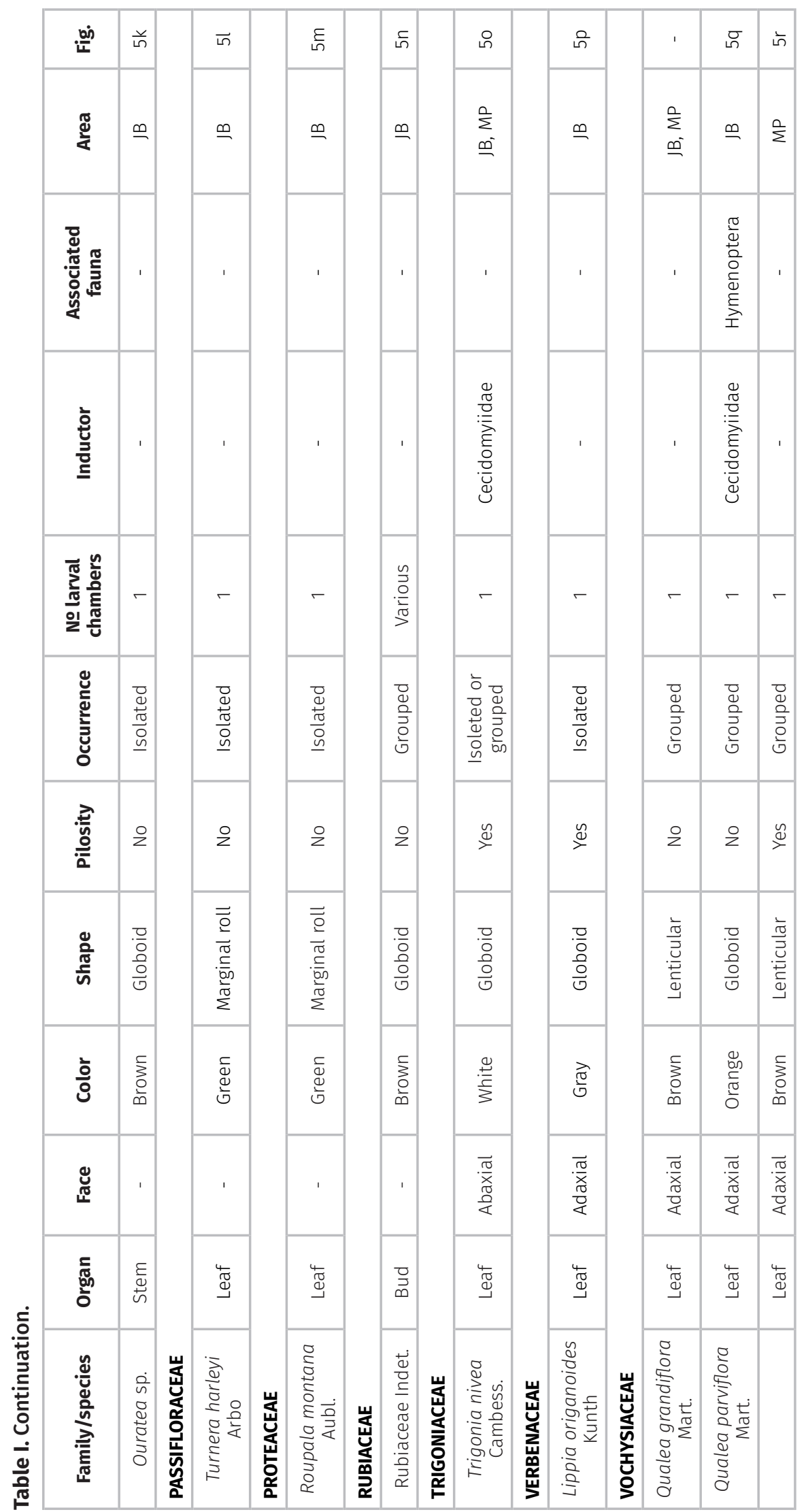

An Acad Bras Cienc (2021) 93(Suppl. 3) e20201442 9| 19 

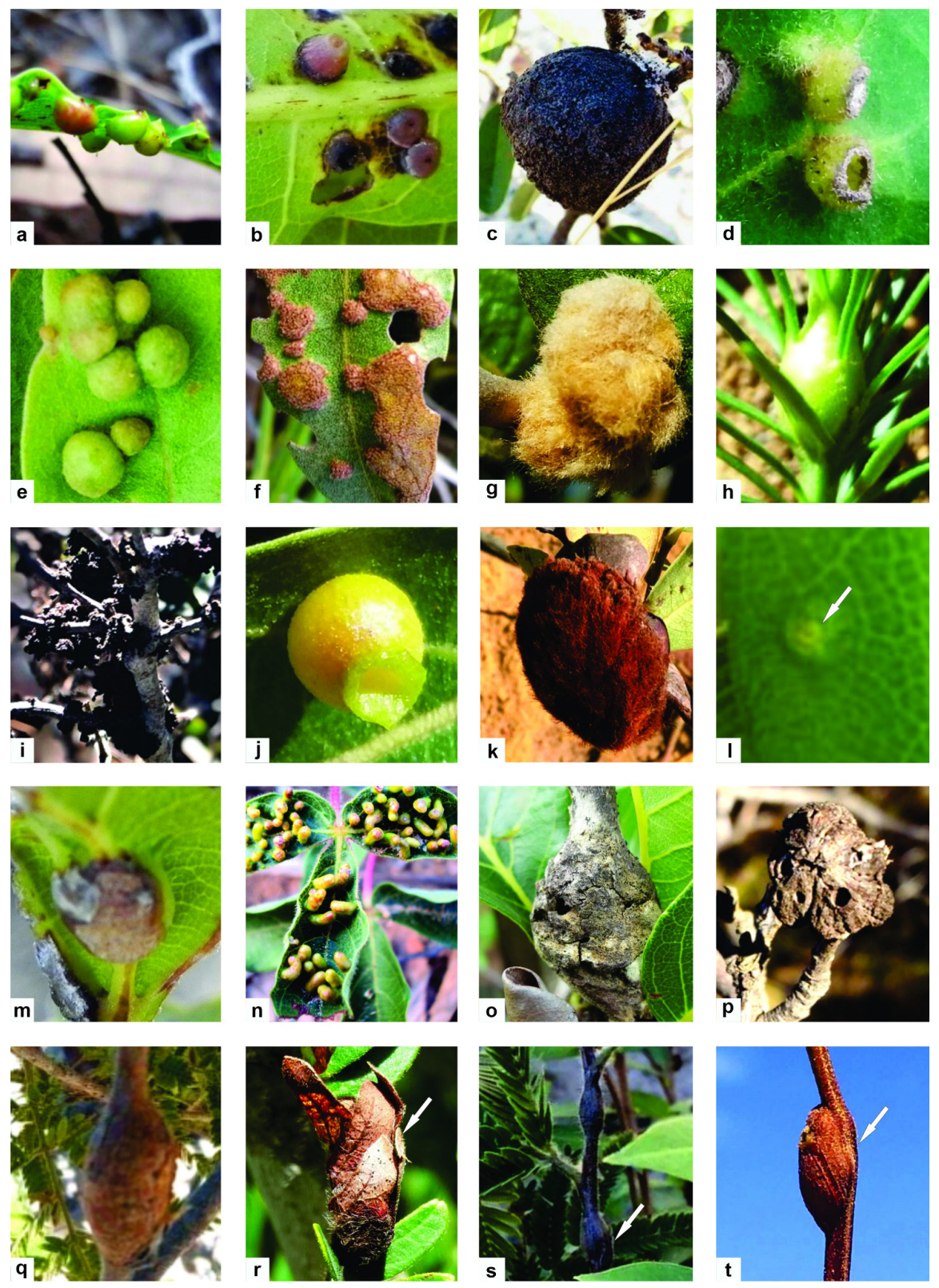

Figure 1. Insect galls in two areas of Cerrado sensu stricto vegetation in Caetité, Bahia State, Brazil. a-b. Anacardium humile; c. Annonaceae Indet. d. Annona leptopetala; e-f. Duguetia furfuracea; $g$. Moquiniastrum paniculatum; h. Pseudobrickellia brasiliensis; i. Maytenus sp.; j. Combretum leprosum; $\mathrm{k}$. Erythroxylum suberosum; l. Erythroxylum stipulosum; m. Alchornea tiliifolia; $\mathrm{n}$. Manihot tripartita; o.Ocotea lancifolia; $p$-r. Calliandra dysantha; s. Calliandra macrocalyx; t. Mimosa gemmulata. was Copaifera langsdorffii Desf ( $\mathrm{n}=12$ gall morphotypes; Figs. 2m-o, 2q-t, 3a-e).

On the MP archaeological site, 40 gall morphotypes were found on 29 plant species belonging to 18 genera and 13 families (Table I). The plant families that hosted the greatest richness of gall morphotypes were Leguminosae and Malpighiaceae ( $n=16$ and $n=8$, respectively). The plant genera with the greatest richness of galls were Copaifera L. ( $\mathrm{n}=10$ gall morphotypes, $\mathrm{n}=2$ host species) and Byrsonima Rich. ex Kunth ( $n=5$ gall morphotypes, $n=3$ host species). The super-host species with the greatest richness of gall morphotypes was Copaifera langsdorffii (n = 9 gall morphotypes; Figs. 2m-n, p, r, t, 3a, f-h).

The galls were found only on vegetative organs: leaves ( $n=88,57$ in JB and 32 in MP), stems ( $n=23,15$ in JB and eight in MP), and buds 

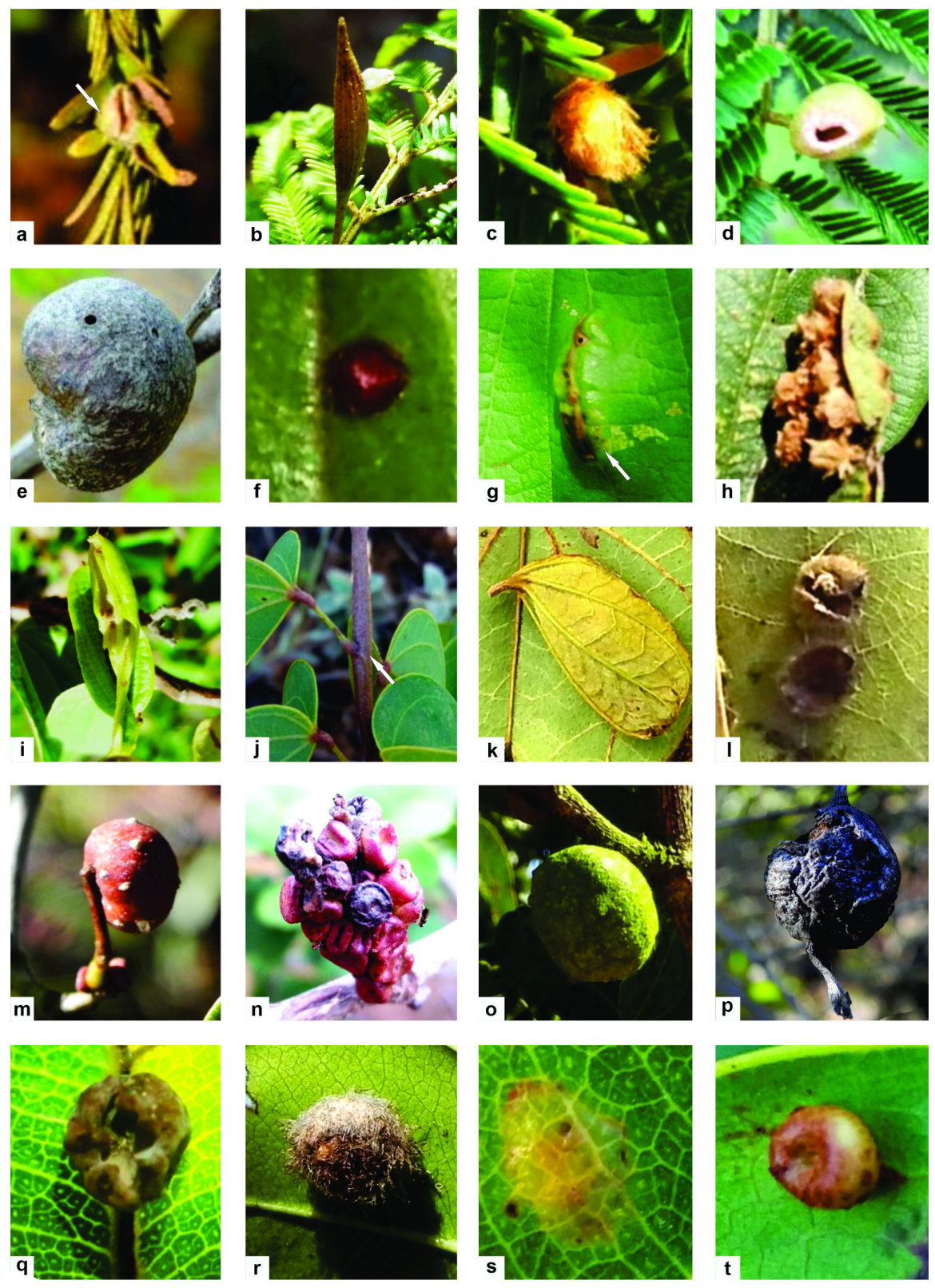
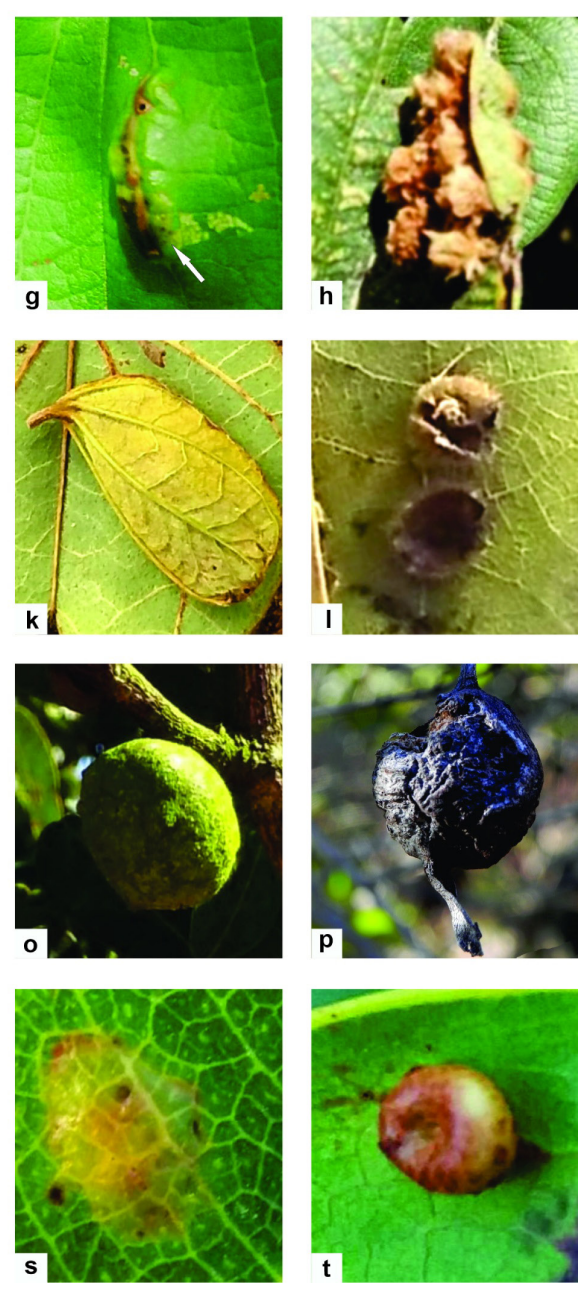

Figure 2. Insect galls in two areas of Cerrado sensu stricto vegetation in Caetité, Bahia State, Brazil. a-d. Mimosa gemmulata; e. Senegalia langsdorffi; f. Senna cana var. cana; g-i. Bauhinia acuruana; $j-l$. Bauhinia pulchella; m-t. Copaifera langsdorffii.
( $n=4$ in JB). Most gall morphotypes occurred on a single plant organ; only one gall morphotype induced on Moquiniastrum paniculatum (Less.) G. Sancho (Asteraceae) was observed on both stems and leaves (Table I, Fig. 1g).

The most frequent gall shapes were globoid ( $n=54,32$ in JB and 22 in MP), lenticular ( $n=$ 23, 15 in JB and 8 in MP), and fusiform ( $n=12$, 7 in JB and 5 in MP). Most galls were glabrous
( $n=101,67$ in JB and 34 in MP), unilocular ( $n=$ 94,64 in JB and 30 in MP), and solitary ( $n=58$, 41 in JB and 17 in MP) or grouped ( $n=55,32$ in $J B$ and 23 in MP). Gall color were green, brown, red, yellow, orange, gray, white, black or purple, being the brown color the most frequent ( $n=$ 44, 26 in JB and 18 in MP). The globoid leaf galls of Combretum leprosum Mart. (Combretaceae) could be green, yellow (Fig. 1j) red, or brown. 
The inducer insects that emerged (from only 33 gall morphotypes) belonged to the orders Diptera, Cecidomyiidae ( $n=27,19$ in $J B$, eight in MP), Lepidoptera $(n=2$ in JB), Thysanoptera $(n=1$ in JB), and Coleoptera $(n=$ 1 in MP). The associated fauna was found in 17 gall morphotypes ( $n=13$ in JB, 10 in MP), and composed by parasitoids (Hymenoptera, $n=9$, 9 in $J B$, four in MP), predators (Araneae, $n=3$, one in $J B$, three in $M P$ ), inquilines (Hemiptera [ $n=2$, one in JB, one in MP] and Thysanoptera [ $n=2$, one in JB, one in MP]), and successors (Formicidae $\mathrm{n}=1$ in JB and MP).

The Sørensen's index values indicated high similarity in host plants between JB farm and MP archaeological site $(S=0.66)$. These localities shared 15 host plant species (Table I). In relation to the morphotypes galls, the similarity is low $(\mathrm{S}=0.36)$, with only 15 gall morphotypes common to both areas, showing that these localities differed in relation to the gall composition species.

\section{DISCUSSION}

The gall richness in JB and MP were high in comparison to other areas with Cerrado sensu stricto vegetation (Araújo et al. 2007, UrsoGuimarães et al. 2003, Coelho et al. 2013, Nogueira et al. 2016), corroborating other studies that indicate that the Cerrado has the richest fauna of galling insects in Brazil (Araújo 2018, Cintra et al. 2020).

The plant families with the greatest numbers of species in a given area generally correspond to those that host the greatest gall richness (Araújo et al. 2014, Santos-Silva \& Araújo 2020). Leguminosae, Malpighiaceae and Myrtaceae were the main host plant families of galls in the two Cerrado s.s. areas, a result similar to reports of other inventories realized in the Bahia Cerrado (Nogueira et al. 2016, Vieira et al. 2018). Those botanical families together with Asteraceae and Melastomataceae hosting a rich diversity of gall-inducing insects in the Cerrado ( $n=468$ gall morphotypes), with many species bearing galls ( $n=263$ spp.) (Cintra et al. 2020).

In addition to those super-host plant families, gall richness can be influenced by presence of super-host species that hosted more than double the number of gall morphotypes compared to other species (Mendonça 2007, Araújo et al. 2013), as is evidenced to Copaifera langsdorffii is this study. This species is frequent in the study area and is considered one of the principal super-hosts of galls in the Brazilian Cerrado (Costa et al. 2010, Lima \& Calado 2018, Fagundes et al. 2018). According to Araújo et al. (2013), the plant species richness explains more than $49 \%$ of the gall inducing insect species richness in the Neotropical savannas, because their important role in the attraction of natural enemies and adaptive radiation of galling species.

Galls induced principally on leaves followed by stems is similar to the patterns seen throughout Brazil (Fernandes \& Negreiros 2006, Santos et al. 2011b, 2012a, Toma \& Mendonça Junior 2013, Santana \& Isaias 2014, Nogueira et al. 2016, Silva et al. 2018). Leaves are considered to be more plastic host organs as compared to stems (Isaias et al. 2013). Additionally, it has been proposed that greater incidence of leaf galls reflects higher levels of nutritional reserves available in the leaves and their photosynthetic capacities (Castro et al. 2012). Almost all galls occurred on a single plant organ, confirming the gall-inducer specificity for the host plant organ (Dreger- Jauffret \& Shorthouse 1992).

Globoid, lenticular, and fusiform galls are generally the predominate shapes in inventories in the Neotropical region (e.g., Urso-Guimarães et al. 2003, Maia 2013a, b, Costa et al. 2014b, Vieira 

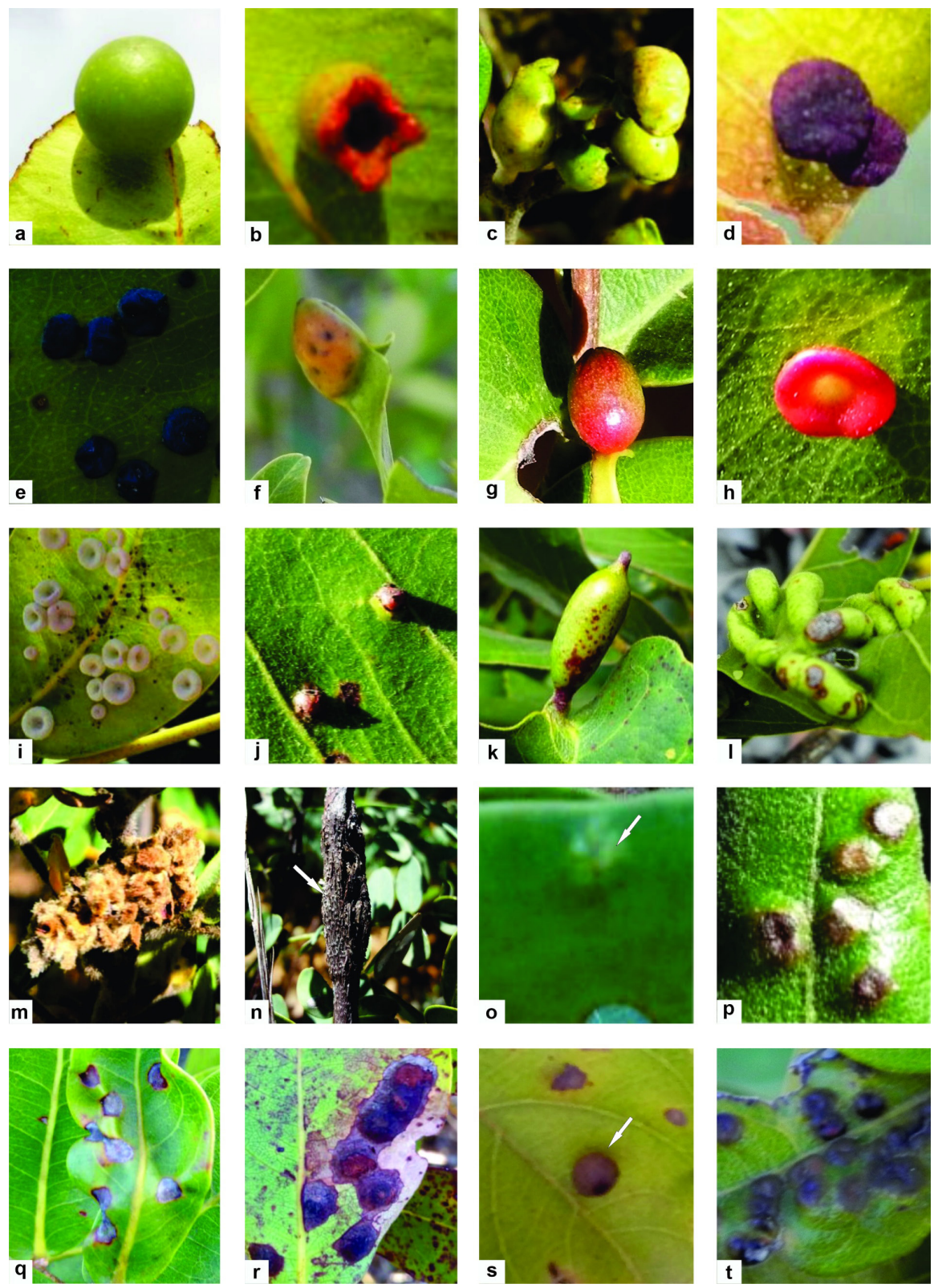
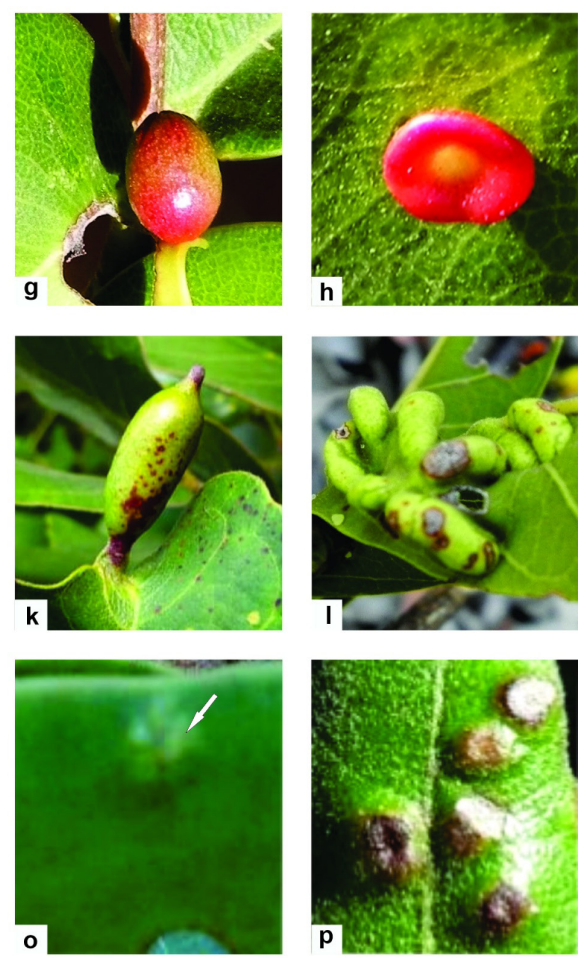
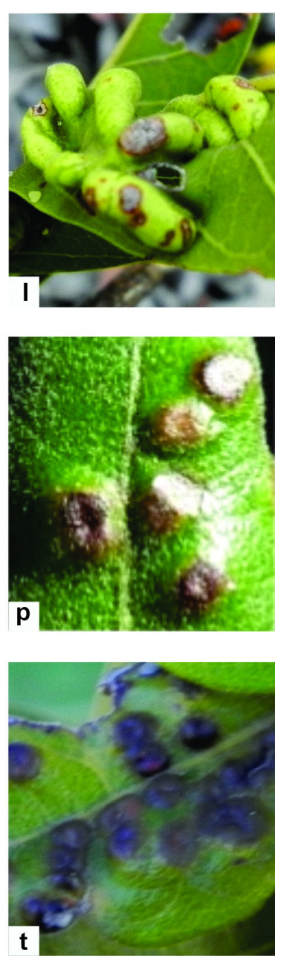

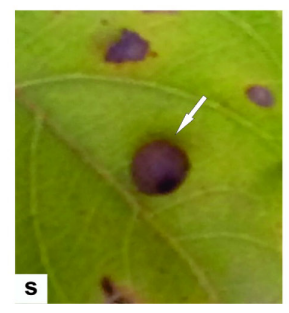

Figure 3. Insect galls in two areas of Cerrado sensu stricto vegetation in Caetité, Bahia State, Brazil. a-h. Copaifera langsdorffi; i. Copaifera luetzelburgii; j-k.Hymenaea courbaril; l. Andira parvifolia; m. Dalbergia acuta; $\mathbf{n}$. Dalbergia miscolobium; o. Struthanthus sp.; p-q. Malpighiaceae Indet. 1; r. Malpighiaceae Indet. 2; s. Malpighiaceae Indet. 3.; t. Malpighiaceae Indet. 4. et al. 2018, Silva et al. 2018). The association between gall morphotypes and host organs was observed, with most globoid and lenticular galls being found on leaves, while fusiform shapes were most common on host stems. Stem galls are often elongated and/or appressed to that organ, following length (Isaias et al. 2013, Ferreira \& Isaias 2013, Santana \& Isaias 2014). While leaf galls tend to distributed equally over the leaf blade, making maximum and efficient use of that surface as well as its total volume, which the cell expansions can occur in all directions (Isaias et al. 2013).

The gall color were predominantly brown, similar to the colors reported in Cerrado and Caatinga-Cerrado transition sites in the Serra Geral at Caetité (Nogueira et al. 2016). The brown color of leaf galls often indicates their final stages of development (Carneiro et al. 2017). In addition, the gall color can also indicate the 

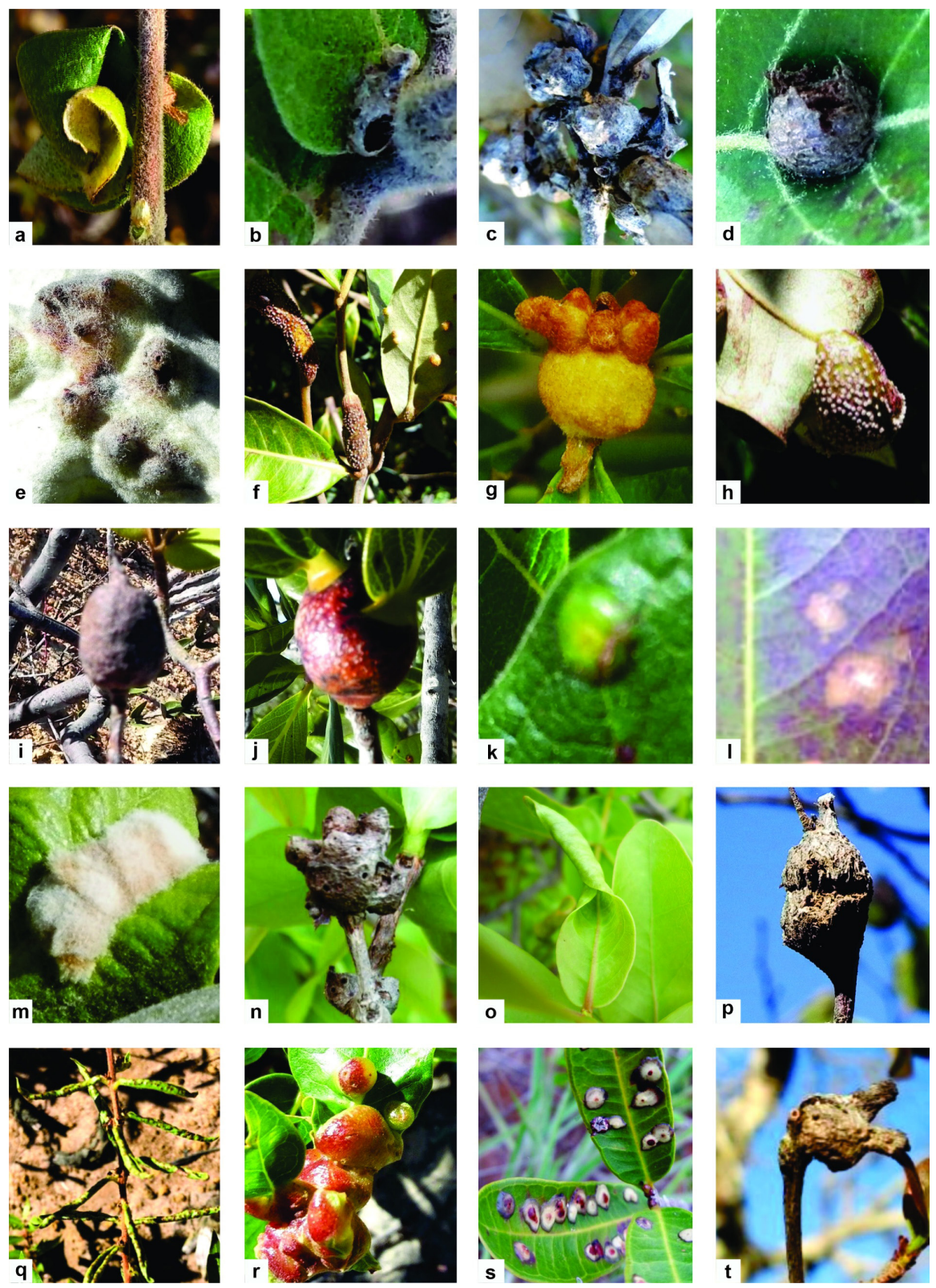

Figure 4. Insect galls in two areas of Cerrado sensu stricto vegetation in Caetité, Bahia State, Brazil. a-b. Banisteriopsis malifolia; c. Byrsonima correifolia; d-e. Byrsonima gardneriana; f-h. Byrsonima sericea;

i. Byrsonima sp.; j. Diospyros sp. k. Diplopterys pubipetala; l. Stigmaphyllon paralias; m. Helicteres sp.; n-o. Moraceae Indet. 1.; $p$. Myrtaceae Indet. 1; q. Myrtaceae Indet. 2; r. Myrtaceae Indet. 3; s. Eugenia sp.; t. Eugenia dysenterica. presence of parasitoids or aposematism (Inbar et al. 2010, Dias et al. 2013). As such, gall color variations represent an open field for research, and their colors may serve to alert predators to the possible presence of toxic compounds (Inbar et al. 2010).

In spite of numerous inventories of galls in different Brazilian ecosystems, our lack of knowledge about the inducer insect species is a barrier to more comprehensive studies
(Melo-Júnior et al. 2018). That situation reflects the difficulty of obtaining specimens of galling insects (including immature and adult phases of both sexes, necessary for species determinations), the prevalence of galls that have already been abandoned by their inducer, our general lack of knowledge of the life histories of the gall inducers, the limited number of taxonomists specialized in gall-inducing groups, and the high mortality rates to the host 

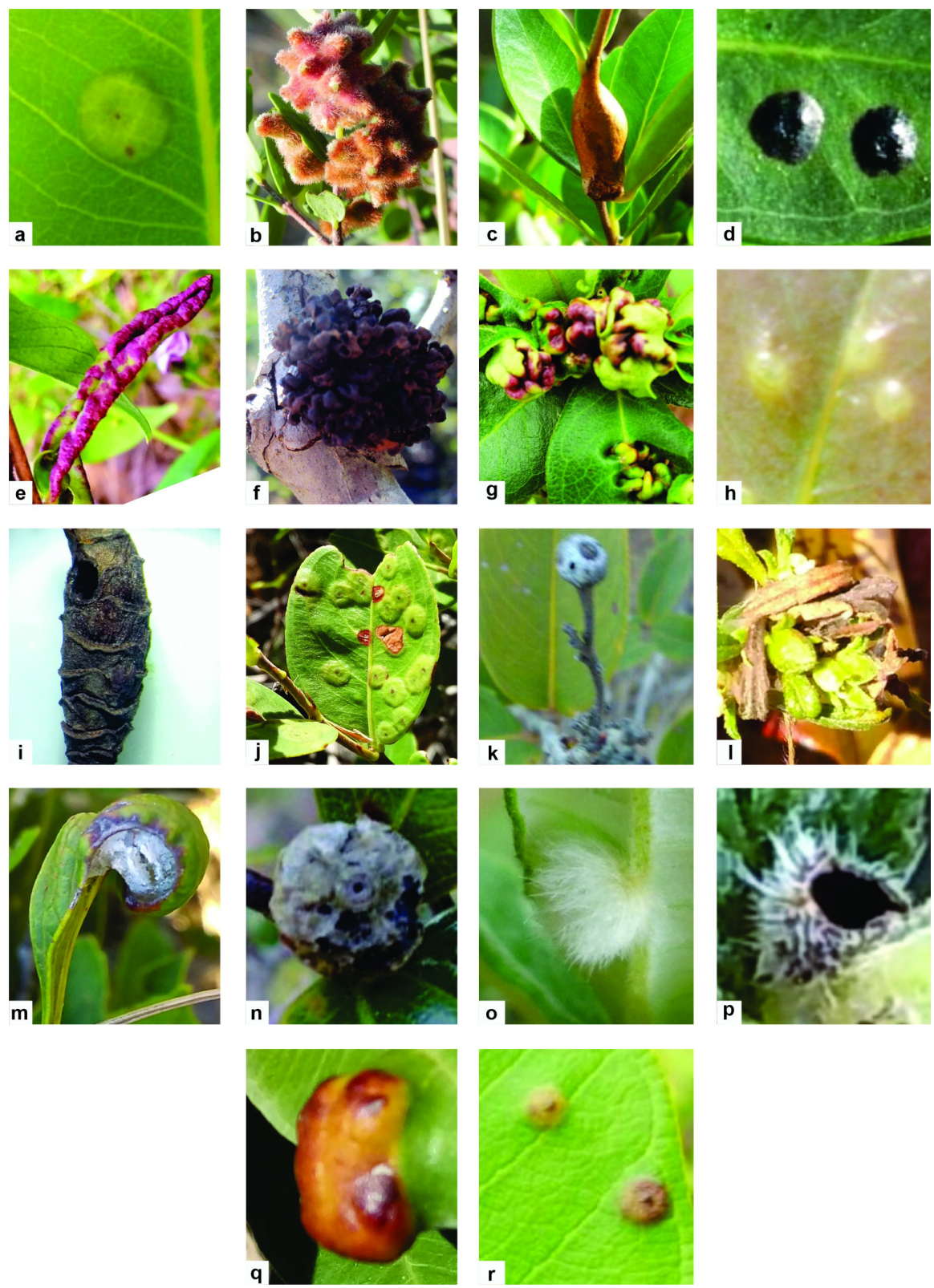

Figure 5. Insect galls in two areas of Cerrado sensu stricto vegetation in Caetité, Bahia State, Brazil. a. Eugenia dysenterica; b. Eugenia lucidifolia; c-e. Eugenia punicifolia; f-g. Myrcia tomentosa; $h$. Guapira laxa; i-j. Ouratea semiserrata; k. Ouratea sp.; l. Turnera harleyi; m. Roupala montana; n. Rubiaceae Indet.; 0 . Trigonia nivea; $\mathrm{p}$. Lippia origanoides; q-r. Qualea parviflora. galler larvae, due the incidence of parasitoids (Espírito-Santo \& Fernandes 2007, Carneiro et al. 2009b, Gagné \& Jaschhof 2017, Melo-Júnior et al. 2018). Of the 98 gall morphotypes recorded in the present work, the insect inducers were identified in only 26 (24 insects to family, one to genus, and just one to species level [Myrciaryiamia admirabilis Maia, 2007, Diptera, Cecidomyiidae]). The Cecidomyiidae (Diptera) were found to be the principal gall inducers, as expected. Cecidomyiidae stands out in the literature as one of the principal gall-inducing groups in the Neotropics (e.g., Carneiro et al. 2009b, Santos et al. 2011a, 2012a, b, Maia 2013a, b, Costa et al. 2014b, Nogueira et al. 2016, Gagné \& Jaschhof 2017, Silva et al. 2018, Santana et al. 2020, Santos-Silva \& Araújo 2020).

The parasitoids were more frequent than inquilines, predators, and successors - another pattern recovered in our results. Plant galls are 
preyed upon by a diverse group of parasitoids belong to Hymenoptera. These organisms frequently cause high mortality rates to the host gall-inducing larvae (Weis \& Abrahamson 1985, Price \& Clancy 1986). The parasitoid community associated with host plant from Cerrado vegetation had already been widely reported in other inventories (Maia 2001, Maia \& Fernandes 2004).

Our results add evidences to the plant diversity hypothesis, the leaves as mainly host organ, the predominance of globoid gall, and Cecidomyiidae as one of the principal gallinducing groups in Cerrado of Caetité, Bahia. We report new species of host plants and new gall morphotypes for the Brazilian Cerrado, which contribute to the understanding of the distribution and diversity of galls in the Cerrado. The great richness of gall morphotypes and host plants found and the high incidence of galling insects of uncertain taxonomic identities indicate that many of them must represent new records for the region, which suggests for the conservation of the two study areas. Additionally, the galling insect guild and the host plant composition varied among Cerrado localities, evidencing the importance of studying and preserving different areas of the same biomes.

\section{Acknowledgments}

The authors are grateful to Msc. Jamile Jorge Ferreira, Cleiton Couto, and Jaqueline Teixeira for their help with the field collections; to Dr. Luciano Paganucci, Dr. Flávio França, and Dr. Efigênia de Mello at the Universidade Estadual de Feira de Santana (UEFS) for their help in identifying the botanical material; and to the Conselho Nacional de Desenvolvimento Científico e Tecnológico (CNPq 406111/2016-2), the Fundação de Amparo à Pesquisa do Estado da Bahia (FAPESB 9648/2015) and the Universidade do Estado da Bahia (Propublic - 015/2019) for financial support. JSS is grateful to Fundação de Amparo à Pesquisa do Estado do Rio de Janeiro - FAPERJ (E-26/202.501/2019) and CNPq (160015/2019-7) for her research grant.

\section{REFERENCES}

ALVARES CA, STAPE JL, SENTELHAS PC, GONÇALVES JLM \& SPAROVEK G. 2013. Köppen's climate classification map for Brazil. Meteorol Z 22: 711-728.

ALVES JES. 2008. Mapeamento Geológico e Análise Estrutural Multiescalar do Cinturão de Dobramentos e Cavalgamentos da Serra do Espinhaço Setentrional, Caetité, Bahia. Monografia (Bacharelado em Geologia) - Instituto de Geociências, Universidade Federal da Bahia, Salvador, 45 p. (Unpublished).

APG IV. 2016. An update of the Angiosperm Phylogeny Group classification for the orders and families of flowering plants: APG IV. Bot J Linn Soc 181: 1-20.

ARAÚJO WS. 2018. 30 years of research o insect galls in Brazil: ascientometric review. Pap Avulsos Zool 58: e20185834.

ARAÚJO WS, CUEVAS-REYES P \& GUILHERME FAG. 2014. Local and regional determinants of galling-insect richness in Neotropical savanna. J Trop Ecol 30: 269-272.

ARAÚJO WS, GOMES-KLEIN VL \& SANTOS BB. 2007. GalhaS Entomógenas Associadas à Vegetação do Parque Estadual da Serra dos Pireneus, Pirenópolis, Goiás, Brasil. R Bras Bioci 5: 45-47.

ARAÚJO WS \& GUILHERME FAG. 2012. Distribuição de insetos galhadores em diferentes formações vegetais e paisagens do cerrado brasileiro. Biosci J 28: 810-819.

ARAÚJO WS, SCARELI-SANTOS C, GUILHERME FAG \& CUEVASREYES P. 2013. Comparing galling insect richness among Neotropical savannas: effects of plant richness, vegetation structure and super-host presence. Biodivers Conserv 22: 1083-1094.

BFG - THE BRAZIL FLORA GROUP. 2018. Brazilian Flora 2020: innovation and collaboration to meet Target 1 of the Global Strategy for Plant Conservation (GSPC). Rodriguésia 69: 1513-1527.

BFG - THE BRAZIL FLORA GROUP. 2021. Brazilian Flora 2020 project - Projeto Flora do Brasil 2020. v393.274. Instituto de Pesquisas Jardim Botanico do Rio de Janeiro. Dataset/ Checklist. doi:10.15468/1mtkaw.

BRITO GP, COSTA EC, CARVALHO-FERNANDES SP \& SANTOSSILVA J. 2018. Riqueza de galhas de insetos em sítios de Caatinga com diferentes graus de impacto antropogênico, Bahia, Brasil. Iheringia, Sér Zool 108: 1-9.

CARNEIRO RG, ISAIAS R, MOREIRA AS \& OLIVEIRA DC. 2017. Reacquisition of new meristematic sites determines the development of a new organ, the Cecidomyiidae gall 
on Copaifera langsdorffii Desf. (Fabaceae). Front Plant Sci 8: 1622.

CARNEIRO MAA, BORGES RAX, ARAÚJO APA \& FERNANDES GW. 2009a. Insetos indutores de galhas da porção Sul da Cadeia do Espinhaço Minas Gerais, Brasil. R Bras Entomol 53: 570-592.

CARNEIRO MAA, BRANCO, CSA, BRAGA CED, ALMADA ED, COSTA MBM, MAIA, VC \& FERNANDES GW. 2009b. Are gall midge species (Diptera, Cecidomyiidae) host-plant specialists? R Bras Entomol 53: 65-378.

CASTRO AC, OLIVEIRA DC, MOREIRA ASFP, LEMOS-FILHO JP \& ISAIAS RMS. 2012. Source-sink relationship and photosynthesis in the horn-shaped gall and its host plant Copaifera langsdorffii Desf. (Fabaceae). S Afr J Bot 83: 121-126.

CINTRA CF ET AL. 2020. Plant-galling insect interactions: a dataset of host plants and their gall-inducing insects for the Cerrado.Ecology: e03149.

COELHO MS, ALMADA ED, FERNANDES GW, CARNEIRO MAA, SANTOS RM, QUINTINO AV \& SANCHEZ-AZOFEIFA A. 2009. Gall inducing arthropods from a seasonally dry tropical forest in Serra do Cipó, Brazil. Rev BrasEntomol 53: 404-414.

COELHO MS, CARNEIRO MAA, BRANCO C \& FERNANDES GW. 2013. Insetos indutores de galhas da Serra do Cabral, Minas Gerais, Brasil. Biota Neotrop 13: 102-109.

COSTA EC, CARVALHO-FERNANDES SP \& SANTOS-SILVA J. $2014 \mathrm{a}$. Galhas entomógenas associadas à Leguminosae do entorno do riacho Jatobá, Caeitité, Bahia, Brasil. R Bras Bioci 12: 115-120.

COSTA EC, CARVALHO-FERNANDES SP \& SANTOS-SILVA J. $2014 \mathrm{~b}$. Galhas de insetos em uma área de transição caatingacerrado no Nordeste do Brasil. Sitientibus Ser Ci Biol 14: $1-9$.

COSTA FV, FAGUNDES M \& NEVES FS. 2010. Arquitetura da planta e diversidade de galhas associadas a Copaifera langsdorffii (Fabaceae). Austral Ecol 20: 9-17.

DIAS GG, MOREIRA GRP, FERREIRA BG \& ISAIAS RMS. 2013. Why do the galls induced by Calophya duvauae Scott on Schinus polygamus (Cav.) Cabrera (Anacardiaceae) change colors? Biochem Syst Ecol 48: 111-122.

DNPM - DEPARTAMENTO NACIONAL DE PESQUISA MINERAL. 2014. Salvador: DNPM. http://www.dnpm.gov.br/acesso-ainformacao / legislacao/portarias-do-diretor-geral-dodnpm/portarias-do-diretor-geral/portaria-no-144-em03-05-2007-do-diretor-geral-do-dnpm. (Accessed March 1, 2018).

DREGER-JAUFFRET F \& SHORTHOUSE JD. 1992. Diversity of gall-inducing insects and their galls. In: Shorthouse JD
\& Rohfritsch O (Eds), Biology of Insect-Induced Galls. Oxford University Press, Oxford, England, p. 285.

ESPIRITO-SANTO MM \& FERNANDES GW. 2007. How Many Species of Gall-inducing insects are there on earth, and where are they? Ann Entomol Soc Am 100: 95-99.

FAGUNDES M, XAVIER RCF, FARIA ML, CUEVAS-REYES P, LOPES LG \& REIS-JUNIOR R. 2018. Plant phenological asynchrony and community structure of gall-inducing insects in a superhost tropical tree species. Ecol Evol 8: 10687-10697.

FALEIRO FG. 2015. Ecossistema do cerrado na Bahia: possibilidades de desenvolvimento agrícola sustentável e sugestões de linhas de pesquisa. In: Baiardi A (Ed), Potencial de agricultura sustentável na Bahia: possibilidades e sugestões de linhas de pesquisa por ecossistemas. Salvador: EDUFBA.

FERNANDES GW \& NEGREIROS D. 2006. A comunidade de insetos galhadores de RPPN Fazenda Bulcão, Aimorés, Minas Gerais, Brasil. Lundiana 7: 111-120.

FERREIRA BG \& ISAIAS RMS. 2013. Developmental stem anatomy and tissue redifferentiation induced by a galling Lepidoptera on Marcetia taxifolia (Melastomataceae). Botany 91: 752-760.

FURTADO, AG, URBANETZ C, VIANEI LK, SÁ R, CANDIDO PC, ANJOS LA, CERQUEIRA MR \& RAIMUNDO RLG. 2003. A frequência de Plantas Herviboras difere entre uma área de Cerrado e uma área Adjacente com Pertubação Antrópica? UNICAMP. https://www2.ib.unicamp.br/profs/fsantos/ relatorios/ne211r4a2003.pdf. (Accessed April 8, 2018).

GAGNÉ RJ \& JASCHHOFM. 2017. A Catalog of the Cecidomyiidae (Diptera) of the world. $4^{\text {th }}$ ed., 762 p. https://www.ars. usda.gov/ARSUserFiles/80420580/Gagne_2017_World_ Cat_4th_ed.pdf (Accessed September 2, 2020).

GONÇALVES-ALVIM SJ \& FERNANDES GW. 2001. Biodiversity of gallinginsects: historical, community and habitat effects in four Neotropical savannas. Biod Conserv 10: 79-98.

HARLEY RM. 1995. Introduction. 1-853p. In: Stannard BL, Harvey YB \& Harley RM (Eds), Flora of the Pico das Almas, Chapada Diamantina - Bahia, Brazil. Kew, Royal Botanic Gardens.

HARLEY RM ET AL. 2005. Cerrado, p. 121-152. In: Juncá FA, Funch L \& Rocha W (Eds), Biodiversidade e conservação da Chapada Diamantina. Brasília: MMA.

IBGE - INSTITUTO BRASILEIRO DE GEOGRAFIA E ESTATÍSTICA. 2015. Manuais técnicos em geociências: manual técnico da vegetação brasileira. Sistema fitogeográfico. Inventário das formações florestais e campestres. Técnicas e manejo de coleções botânicas e procedimentos para mapeamentos. 2a ed., IBGE, Rio de Janeiro, 271 p. 
INBAR M, IZHAKI I, KOPLOVICH A, LUPO, I, SILANIKOVE N, GLASSER T GERCHMAN Y, PEREVOLOTSKY A \& LEV-YADUN S. 2010. Why do many galls have conspicuous colors? A new hipothesis. Arth Plant Int 4: 1-6.

ISAIAS RMS, CARNEIRO RGS, OLIVEIRA DC \& SANTOS JC. 2013. Illustrated and Annotated Checklist of Brazilian Gall Morphotypes. Neotrop Entomol 42: 230-239.

JULIÃO GR, AMARAL ME \& FERNANDES GW. 2002. Galhas de insetos e suas plantas hospedeiras no Pantanal SulMatogrossense. Naturalia 27: 47-74.

JULIAO GR, FERNANDES W, NEGREIROS D, BEDÊ L \& ARAÚJO RC. 2005. Insetos galhadores associados a duas espécies de plantas invasoras de áreasurbanas e peri-urbanas. $\mathrm{R}$ Bras Entomol 49: 97-106.

KLINK CA \& MACHADO RB. 2005. Conservation of the brazilian cerrado. Conserv Biol 19: 707-713.

LIMA VP \& CALADO D. 2018. Morphological characterization of insect galls and new records of associated invertebrates in a Cerrado area in Bahia State. Braz J Biol 78: 636-643.

LPWG - THE LEGUME PHYLOGENY WORKING GROUP. 2017. A community-endorsed phylogenetic classification of the Leguminosae, including a new densely-sampled phylogeny of the family. Taxon 66: 44-77.

MAIA VC. 2001. The gall midges (Diptera, Cecidomyiidae) from three restingas of Rio de Janeiro State, Brazil. Rev Bras Zool 18: 583-629.

MAIA VC \& FERNANDES GW. 2004. Insect galls from Serra de São José (Tiradentes, MG, Brazil). Braz J Biol 64: 423-445.

MAIA VC. 2013a. Insect galls from restingas of Southeastern Brazil, with newrecords. Biota Neotrop 13: 183-209.

MAIA VC. 2013b. Insect galls of São Tomé das Letras (MG, Brazil). Biota Neotrop 13: 164-189.

MELO-JÚNIOR JCF, ISAIAS RMS, BOEGER MRT, ARRIOLA IA \& MATILDE-SILVA M. 2018. Diversidade de galhadores nas restingas do ecossistema Babitonga, Santa Catarina, Brasil. Revista Cepsul - Biodiversidade e Conservação Marinha 7: eb2018003.

MENDONÇA MS. 2007. Plant diversity and galling arthropod diversity searching for taxonomic patterns in an animalplant interaction in the neotropics. BolSoc Argent Bot 42: 347-357.

MMA. 2015. Mapeamento do uso e cobertura do Cerrado: Projeto Terra Class Cerrado. Available at: http:// www.mma.gov.br/images/arquivo/80049/Cerrado/ publicacoes/Livro\%20 EMBRAPA-WEB-1-TerraClass $\% 20$ Cerrado.pdf (Accessed April 8, 2018).
NOGUEIRA, RM, COSTA EC, CARVALHO-FERNANDES SP \& SANTOSSILVA J. 2016. Insect galls from Serra Geral, Caetité, BA, Brazil. Biota Neotrop 16:e20150035.

OLIVEIRA JC. 2009. Viabilidade de espécies galhadoras (Diptera, Cecidomyiidae) e parasitóides (Hymenoptera) associadas à Guapira opposita (Vell.) (Nyctaginaceae) como bioindicadores da qualidade ambiental. Tese de Doutorado, Universidade Federal do Rio de Janeiro, Rio de Janeiro.

OLIVEIRA JC \& MAIA VC. 2005. Ocorrência e caracterização de galhas de insetos na restinga de Grumari (Rio de Janeiro, RJ, Brasil). Arq Mus Nac 63: 669-675.

PRICE PW \& CLANCY KM. 1986. Interactions among three trophic levels: gallsize and parasitoid attack. Ecology 67: 1593-1600.

PRICE PW, FERNANDES GW \& WARING GL. 1987. Adaptive Nature ofInsect Galls. Environ Entomol 16: 15-24.

PRUDENTE TA, ANJOS JASA \& SOARES AGL. 2017. Geração de energia elétrica por fonte eólica. Bahia Análise \& Dados, Salvador, 27: 28-48, como atua o licenciamento ambiental no estado da Bahia, EISSN 2595-2064.

RIBEIRO SP \& FERNANDES GW. 2000. Interações entre insetos e plantas no Cerrado: teoria e hipóteses de trabalho. In: Martins RP, Lewinsohn TM \& Barbeitos MS (Eds), Ecologia e comportamento de insetos. Oecologia Brasiliensis, Rio de Janeiro, Brasil, p. 299-320.

RODRIGUES OF, SILVA JJF \& NUNES PIC. 2017. Parecer da Nascente do Riacho Pedra de Ferro nos municípios de Caetité e Pindaí-Bahia. II Simpósio Bahiano de Geografia Agrária: entre a teoria e prática, articulações e resistências. Julho 03-05, Salvador, Bahia, p. 1-12.

SANTANA AP \& ISAIAS RMS. 2014. Galling insects are bioindicators of environmental quality in a Conservation Unit. Acta Bot Bras 28: 594-608.

SANTANA CAGS, COSTA EC, CARVALHO-FERNANDES S \& SANTOSSILVA J. 2020. Insect galls and their host plants in gallery forest in Bahia State, Brazil. Braz J Bot 43: 989-998.

SANTOS BB, RIBEIRO BA, SILVA TM \& ARAÚJO WS. 2012a. Galhas de insetos em uma área de cerrado sentido restrito na região semi-urbana de Caldas Novas (Goiás, Brasil). Rev Bras Bioci 10: 439-445.

SANTOS JC, ALMEIDA-CORTEZ IS \& FERNANDES GW. 2011 a. Diversity of gall-inducing insects in the high altitude wetland forests in Pernambuco, Northeast Brasil. Braz J Biol 71: 47-56. 
SANTOS JC, ALMEIDA-CORTEZ IS \& FERNANDES GW. 2011b. Richness of gall-inducing insects in the tropical dry forest (caatinga) of Pernambuco. Rev Bras Ent 55: 45-54.

SANTOS JC, ALMEIDA-CORTEZ JS \& FERNANDES GW. 2012b. Gall-inducing insects from Atlantic forest of Pernambuco, northeastern Brazil. Biota Neotrop 12: 197-213.

SANTOS-SILVA J \& ARAÚJO TJ 2020. Are Fabaceae the principal super-hosts of galls in Brazil? An Acad Bras Cienc 92: e20181115.

SHORTHOUSE, JD, WOOL D \& RAMAN A. 2005. Gall-inducing insects - Nature's most sophisticated herbivores. Basic Appl Ecol 6: 407-411.

SILVA AF, NOGUEIRA RM, COSTA EC, CARVALHO-FERNANDES SP \& SANTOS-SILVA J. 2018. Occurrence and characterization of entomogenic galls in an area of Cerrado sensu stricto and Gallery forest of the state of Bahia, Brazil. An Acad Bras Cienc 90: 2903-2919.

S $\varnothing$ RENSEN TA. 1948. A method of establishing groups of equal amplitude in plant sociology based on similarity of species content, and its application to analyses of the vegetation on Danish commons. Kongelige Danske videnskabernes selskab. Biol Skr 5: 1-34.

STONE GN \& SCHÖNROGGE N. 2003.The adaptive significance of insect gallmorphology. Trends Ecol Evol 18: 512-522.

TOMA TSP \& MENDONÇA-JÚNIOR MS. 2013. Gall-inducing insects of an Araucaria Forest in Southern Brazil. Rev Bras Entomol 57: 225-233.

URSO-GUIMARÃES MVC, SCARELI-SANTOS C \& BONIFÁCIO-SILVA A. 2003. Occurrence and characterization of entomogen galls in plants from natural vegetation areas in Delfinópolis, MG, Brazil. Braz J Biol 63: 705-715.

VIEIRA LG, NOGUEIRA RM, COSTA EC, CARVALHO- FERNANDES SP \& SANTOS-SILVA J. 2018. Insect galls in rupestrian field and cerrado stricto sensuvegetation in Caetité, Bahia, Brasil. Biota Neotrop 18: e20170402.

WEIS AE \& ABRAHAMSON WG. 1985. Potencial selective pressures by parasitoids on plant-herbivore interaction. Ecology 66: 1261-1269.

\section{How to cite}

CAMPOS GBD, COSTA EC, SANTOS DLS, CARVALHO-FERNANDES SP \& SANTOS-SILVA J. 2021. Insect galls and associated fauna in two areas of Cerrado sensu stricto in the State of Bahia, Brazil. An Acad Bras Cienc 93: e20201442. DOI 10.1590/0001-3765202120201442.

\section{GABRIELA B.D. CAMPOS}

https://orcid.org/0000-0002-5655-0066

ELAINE C. COSTA ${ }^{2}$

https://orcid.org/0000-0001-6625-7595

DÉBORA L.S. SANTOS ${ }^{3}$

https://orcid.org/0000-0001-5022-7666

SHEILA P. CARVALHO-FERNANDES ${ }^{4}$

https://orcid.org/0000-0003-0754-6594

\section{JULIANA SANTOS-SILVA ${ }^{1}$}

https://orcid.org/0000-0003-0134-3438

${ }^{1}$ Universidade do Estado da Bahia, Programa de Pós-graduação em Biodiversidade Vegetal, Departamento de Educação-DEDC, Rua da Gangorra, 503, CHESF, 48608-240 Paulo Afonso, BA, Brazil

${ }^{2}$ Universidade Federal de Minas Gerais, Programa de Pósgraduação em Biologia Vegetal, Departamento de Botânica, Instituto de Ciências Biológicas, Avenida Antônio Carlos, 6627, Pampulha, 31270-901 Belo Horizonte, MG, Brazil

${ }^{3}$ Universidade do Estado da Bahia, Departamento de Ciências Humanas - DCH, Avenida Contorno, s/n, Centro, 46400-000 Caetité, BA, Brazil

${ }^{4}$ Universidade Federal do Riode Janeiro/UFRJ, Departamento de Entomologia, Museu Nacional, Quinta da Boa Vista, s/n, São Cristóvão, 20940-040 Rio de Janeiro, RJ, Brazil

Correspondence to: Juliana Santos-Silva

E-mail:jussilva@uneb.br

\section{Author contributions}

Gabriela Campos contributed to data collection and to manuscript preparation; Elaine Costa contributed to data analysis and interpretation and to manuscript preparation; Débora Santos contributed to data collection; Sheila CarvalhoFernandes contributed to data analysis and interpretation; Juliana Santos contributed in the concept and design of the study and acquisition of the financial support for the project leading to this publication.

\section{(cc) BY}

